\title{
Numerical simulations of single and binary black holes in scalar-tensor theories: circumventing the no-hair theorem
}

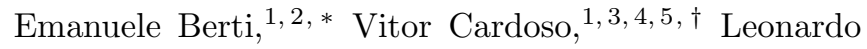

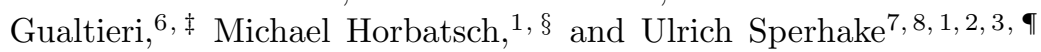 \\ ${ }^{1}$ Department of Physics and Astronomy, The University of Mississippi, University, MS 386r7, USA \\ ${ }^{2}$ California Institute of Technology, Pasadena, CA 91109, USA \\ ${ }^{3}$ CENTRA, Departamento de Física, Instituto Superior Técnico, \\ Universidade Técnica de Lisboa - UTL, Av. Rovisco Pais 1, 1049 Lisboa, Portugal \\ ${ }^{4}$ Perimeter Institute for Theoretical Physics Waterloo, Ontario N2J 2W9, Canada \\ ${ }^{5}$ Faculdade de Física, Universidade Federal do Pará, 66075-110, Belém, Pará, Brazil \\ ${ }^{6}$ Dipartimento di Fisica, Università di Roma "Sapienza" ES Sezione, \\ INFN Roma1, P.A. Moro 5, 00185, Roma, Italy \\ ${ }^{7}$ Department of Applied Mathematics and Theoretical Physics, Centre for Mathematical Sciences, \\ University of Cambridge, Wilberforce Road, Cambridge CB3 0WA, UK \\ ${ }^{8}$ Institut de Ciéncies de l'Espai (CSIC-IEEC), Facultat die Ciéncies, Campus UAB, E-08193 Bellaterra, Spain
}

(Dated: September 7, 2018)

\begin{abstract}
Scalar-tensor theories are a compelling alternative to general relativity and one of the most accepted extensions of Einstein's theory. Black holes in these theories have no hair, but could grow "wigs" supported by time-dependent boundary conditions or spatial gradients. Time-dependent or spatially varying fields lead in general to nontrivial black hole dynamics, with potentially interesting experimental consequences. We carry out a numerical investigation of the dynamics of single and binary black holes in the presence of scalar fields. In particular we study gravitational and scalar radiation from black-hole binaries in a constant scalar-field gradient, and we compare our numerical findings to analytical models. In the single black hole case we find that, after a short transient, the scalar field relaxes to static configurations, in agreement with perturbative calculations. Furthermore we predict analytically (and verify numerically) that accelerated black holes in a scalar-field gradient emit scalar radiation. For a quasicircular black-hole binary, our analytical and numerical calculations show that the dominant component of the scalar radiation is emitted at twice the binary's orbital frequency.
\end{abstract}

\section{INTRODUCTION}

Scalar fields are ubiquitous in physics, either as a proxy for more complex interactions or as fundamental quantities in their own right. For example, one of the best studied modifications to general relativity is scalar-tensor gravity, in which space-time curvature couples to scalar fields (which are sufficiently light to be relevant for astrophysics and/or cosmology). The historical development of this theory goes back to the 1940s, and involves several research groups with different views on the physical interpretation of the scalar degree of freedom [1] 3. In recent times, interest in scalar-tensor gravity has been driven by theoretical attempts to unify gravity with quantum mechanics at high energies and solve the cosmological constant and hierarchy problems, as well as observations of the cosmic microwave background and the highly-anticipated direct detection of gravitational waves [4] 6 .

The simplest version of scalar-tensor gravity is BransDicke theory [7 9], which consists of a single massless

\footnotetext{
* berti@phy.olemiss.edu

$\dagger$ vitor.cardoso@ist.utl.pt

$\ddagger$ Leonardo.Gualtieri@roma1.infn.it

$\S$ mhorbats@olemiss.edu

ฯ sperhake@tapir.caltech.edu
}

scalar $\phi$, whose coupling to curvature is controlled by a dimensionless parameter $\omega_{\mathrm{BD}}$. Generalizations include varying scalar-curvature couplings $\omega(\phi)$ and a scalar potential $V(\phi)$ ("Bergmann-Wagoner" theories [10, 11]) as well as the possibility of multiple interacting scalar fields: see e.g. 4, 12, 13, for comprehensive treatments of the subject. These generalizations (commonly referred to as "scalar-tensor theories") are compelling due to their simplicity, but (perhaps as a consequence) they are also very well constrained observationally. The classic book by Will [14] presents an overview of the subject, and comprehensive reviews of the state of the art in experimental tests of gravitational theories can be found in [15, 16].

In this paper we shall focus, for simplicity, on scalartensor theories involving a single scalar field. In general relativity, because of the conservation of total momentum for isolated systems, gravitational radiation is quadrupolar in nature. A possible smoking gun of scalar-tensor gravity is the existence of dipole radiation, essentially due to violations of the equivalence principle. Solar System experiments and observations of binary pulsar systems place strong constraints on the coupling functions of the theory $12,14,15,17,27$, and other interesting constraints may come from the direct observation of gravitational radiation from binary systems in the near future [21, 28 35]. Despite all of these observational constraints, striking and potentially observable astrophysical 
phenomena are still possible in these theories. Such phenomena include superradiant instabilities (see e.g. [3639] for discussions of this phenomenon in the context of the "string axiverse" scenario [40]) and the related possibility of floating orbits around rotating black holes (BHs) [1].

\section{A. Classical no-hair theorems}

Theoretical studies impose remarkable constraints and limitations on scalar-tensor theories. First of all, the famous BH no-scalar-hair theorems first proved by Hawking 42, Thorne and Dykla [43, and Chase [44] state that stationary $\mathrm{BH}$ solutions in Brans-Dicke theory are the same as those in general relativity. These results have been generalized and expanded upon by many authors. For example, an extension to multiple scalars has been established by Heusler 45, and an extension to Bergmann-Wagoner and $f(R)$ theories has been established by Sotiriou and Faraoni [46]. The no-scalar-hair theorems have also been confirmed by numerical studies of gravitational collapse [47 52. More generally, it has been observed that the Kerr metric is a solution in a wide class of gravity theories 53. However, the fact that stationary vacuum solutions of scalar-tensor theories agree with those of general relativity does not mean that the dynamics of $\mathrm{BHs}$ in these theories must be the same [48, 54,56].

For a comprehensive discussion and literature survey of no-hair theorems, the reader is referred to the reviews of Bekenstein [57 and Chruściel, Costa, and Heusler 58]. Although the literature is vast, there are two basic assumptions lying at the heart of most no-hair theorems. The first is that of stationarity, whose necessity has been demonstrated by Jacobson's "Miracle Hair Growth Formula," a perturbative construction of a hairy $\mathrm{BH}$ with time-dependent scalar boundary conditions 59 .

The second assumption is the truncation of the scalartensor action to second order in the derivative expansion. At this level, the most general action [Eq. (2) in the single-scalar case] is very simple and contains only three terms, whereas scalar-tensor gravity at the fourderivative level is too complicated to be studied in complete generality, and thus, attention is often restricted to particular models.

One such model is quadratically modified gravity, whose action contains all possible terms quadratic in the Riemann tensor, coupled to a scalar. In this theory, BHs have been studied perturbatively, and solutions with scalar hair have been found 6064 . Moreover, in the special case of a scalar coupled to a topological invariant - namely, Einstein-dilaton-Gauss-Bonnet (EDGB) or Dynamical Chern-Simons (DCS) gravity - a no-hair theorem for neutron stars has been established [65. The conclusion here is that spherically-symmetric neutron stars have vanishing scalar monopole moment, but higher-order scalar multipoles need not vanish. How- ever, the presence of derivatives higher than second order in the field equations severely complicates the implementation of numerical simulations.

Although four-derivative actions generically lead to field equations with more than two derivatives, there are some noteworthy exceptions. One is the EinsteinSkyrme system, a nonlinear sigma model with targetspace $S U(2)$, containing a term in the action quartic in scalar derivatives, and admitting linearly-stable BH solutions with scalar hair which have been described numerically in 66 69. Another model with second-order field equations is the galileon [70, which is related to both higher-dimensional Lovelock gravity [71] and massive gravity [72, 73. It satisfies Solar System constraints by means of the Vainshtein mechanism 74, and a nogalileon-hair theorem for spherically-symmetric $\mathrm{BHs}$ has been recently established [75]. Another interesting example is Bergmann-Wagoner scalar-tensor gravity coupled to non-linear electrodynamics, where the non-vanishing trace of the electromagnetic stress-energy tensor enters as a source into the scalar-field equation. This allows for stable asymptotically-flat $\mathrm{BHs}$ with scalar hair, which have been studied numerically in 76 81].

\section{B. A generalized no-hair theorem}

The "classical" no-hair theorems described in the preceeding paragraphs, which are statements about stationary vacuum space-times, have been extended to the context of a BH binary system. Employing the "generalized EIH" formalism developed by Eardley [82, Will and Zaglauer 17] have shown that the leading-order postNewtonian (PN) dynamics of a BH binary in Brans-Dicke theory is indistinguishable from that in general relativity. Recently, this result has been extended to general scalartensor theories in the extreme mass-ratio limit 34 and it has been shown to hold up to $2.5 \mathrm{PN}$ order for generic mass ratio 83. Thus, even a dynamical (vacuum) spacetime with two interacting BHs does not have scalar hair in the PN limit. We can regard this conclusion as a "generalized no-hair theorem."

The generalized no-hair theorem relies on the assumption that the binary system is isolated, in the sense that cosmological and environmental effects (say, due to the galactic background surrounding the $\mathrm{BHs}$ ) are neglected. More precisely, it is assumed that: (1) there is no matter in the system, (2) the scalar field has zero potential, (3) the scalar-tensor action is truncated to second order in the derivative expansion, and (4) the metric is asymptotically flat (in all conformal frames) and the scalar is asymptotically constant.

The vacuum assumption (1) can be relaxed either by considering BHs in astrophysical environments or by considering compact stars, which are affected by the wellknown spontaneous scalarization phenomenon (cf. 84 for recent numerical studies). Other recent numerical studies created a scalar-field "bubble" around the bi- 
nary by using a nonvanishing potential, i.e. relaxing assumption (2). They found that the scalar-field bubble is rapidly accreted by the $\mathrm{BHs}$, modifying the binary dynamics 85]. As for assumption (3), compact binary dynamics in quadratically modified gravity has been studied analytically in a perturbative framework, where one takes the point of view that the model should be considered as an effective low-energy theory 65, 86, 87]. Whether these theories are well posed for numerical evolutions is currently a matter of debate. We will not consider this problem in the present paper, but it is an interesting topic for future research.

Relaxing either assumption (3) or assumption (4) introduces a new length (or time) scale in the $\mathrm{BH}$ binary dynamics. In the case of assumption (3) this scale is determined by the Compton wavelength of a heavy particle, whose square enters into coefficients of four-derivative terms in the action. In the case of assumption (4), the new scale is determined by cosmological and/or galactic effects. A priori, it is not obvious which of these effects is dominant, and thus it is worthwhile to explore both possibilities. To our knowledge, the relaxation of assumption (4) has not been explored in the literature, and it is the main focus of our paper.

Asymptotic flatness of the metric is only an approximation to the dynamics of an astrophysical binary. Observations show that the Universe is expanding on timescales which are very large, but nevertheless finite with respect to astrophysical BH binary evolution. As shown in [59, 88, imposing time-varying boundary conditions endows the BHs in a binary with scalar charge, and therefore the binary can emit dipole scalar radiation. Furthermore, many cosmological models consider the existence of background scalars which can be anchored on matter 89 98. In this case, one can for instance conceive of a $\mathrm{BH}$ binary evolving in the background of a nearly-static but nonuniform scalar field anchored on the galactic matter. The characteristic lengthscale of such a scalar-field profile would be much larger than the binary separation, and therefore it would have the same effect on the dynamical evolution of the binary system as the enforcement of boundary conditions which are not asymptotically flat. Finally, BH dynamics in the background of scalar fields could also be relevant to understanding accretion inside hypothetical supermassive boson stars, where huge scalar-field gradients are expected [99.

Scalar-field gradients can therefore allow us to circumvent the generalized no-hair theorem, i.e., to have a spacetime which contains only BHs and still emits scalar radiation. Indeed, as we shall discuss below [cf. Eq. (28), Section III B 2, in the presence of a spatially-varying scalar-field profile $\varphi(\vec{x})$, a nonrotating $\mathrm{BH}$ of mass $M$ with world-line $(t, \vec{x}(t))$ would have a scalar charge $£^{1}$

$$
\begin{aligned}
Q(t) & =4 M^{2} \frac{d \vec{x}(t)}{d t} \cdot \vec{\nabla} \varphi(\vec{x}(t)) \\
& =8 \pi \sigma M^{2} \frac{d \vec{x}(t)}{d t} \cdot \hat{z}
\end{aligned}
$$

where in the second line we have assumed that the scalarfield gradient is directed along the $z$-axis, and we have parametrized its magnitude by a real parameter $\sigma$. If the $\mathrm{BH}$ is accelerated, or if the scalar gradient is nonuniform, the scalar charge would evolve in time, yielding scalar radiation. As we show in Appendix D for a stellar-mass BH $\left(M=10 M_{\odot}\right)$ moving near the galactic center a typical scalar-field gradient is $M \sigma \sim 10^{-15}$. For a supermassive BH with $M=10^{9} M_{\odot}$ a typical gradient could be as large as $M \sigma \sim 10^{-7}$, comparable in order of magnitude to the numerical simulations presented in this paper.

\section{Executive summary and plan of the paper}

The main goal of this work is to explore the consequences of the presence of a scalar-field gradient, which is equivalent to imposing nontrivial boundary conditions on the dynamics of a $\mathrm{BH}$ binary, and to verify numerically whether, as suggested by Eq. (1), such a setup can allow scalar radiation from a BH binary system in scalartensor theory. Here we present an executive summary of our main results and an outline of the paper.

In Section II we lay out our theoretical framework by introducing generic scalar-tensor theories and presenting the relations that allow us to transform between the Jordan frame (where physical quantities should be computed) and the Einstein frame (where we will perform our calculations). In particular, we show how gravitational radiation in the Jordan frame can be computed from a knowledge of the Newman-Penrose scalars in the Einstein frame.

In Section III we introduce analytical approximations for scalar fields in the background of single and binary BH spacetimes. These approximations are useful to validate (and provide insight into) our numerical simulations. In fact, numerical evolutions of initial data corresponding to a single black hole in a scalar gradient show that, after a short transient, the scalar field relaxes to the static configurations predicted by these perturbative calculations. For a quasicircular black-hole binary, in Section III we show analytically that the dipole component of the scalar radiation is emitted at twice the binary's orbital frequency. This prediction is validated by our numerical

\footnotetext{
1 The scalar charge $Q$ and mass $M$ are Einstein-frame quantities, and geometrical units $G=c=1$ are employed, where $G$ is the Einstein-frame bare gravitational constant. The result quoted here also assumes that the $\mathrm{BH}$ motion relative to the scalar-field profile is "slow", in the sense that $M(d \vec{x} / d t) \cdot \vec{\nabla} \varphi \ll 1$.
} 
simulations, which also show that the dipole component dominates the scalar emission.

In Section [V] we present the details of our numerical implementation. The results of our simulations are discussed and compared with analytical results in Section V] In Section VI we summarize our findings and point out possible directions for future work.

To improve readability, in the Appendices we collect technical material that illustrates various important points of our analysis. Appendix A shows that a BH moving with constant velocity in a uniform scalar-field gradient does not emit scalar radiation. Appendix $\mathrm{B}$ (which is complementary to Section III B 2 collects some lengthy formulas illustrating the structure of gravitational radiation from a $\mathrm{BH}$ binary moving in a scalar-field gradient. In Appendix C we provide explicit expressions for the evolution equations used in our numerical code. In Appendix D we estimate the order of magnitude of the scalar-field gradients expected in scalar-field models of dark matter.

\section{THEORETICAL FRAMEWORK}

We focus on general single-scalar-tensor theories in vacuum with vanishing scalar potential, and at most two derivatives in the action. These theories are equivalent to Einstein's theory extended to include a minimally coupled scalar field with vanishing potential. This statement (which will be clarified below) has a nontrivial consequence: the addition of minimally coupled scalars to Einstein's gravity allows one to study a multitude of scalartensor theories at once. For this reason our simple framework offers an opportunity to take a glimpse at a rather broad spectrum of physics beyond Einstein's theory.

Our starting point is the action of a general scalartensor theory for a single scalar field $\phi$, written as

$S=\int d^{4} x \frac{\sqrt{-g}}{16 \pi G}\left(F(\phi) R-8 \pi G Z(\phi) g^{\mu \nu} \partial_{\mu} \phi \partial_{\nu} \phi-U(\phi)\right)$,

where $R$ is the Ricci scalar associated to the metric $g_{\mu \nu}$, and $F(\phi), Z(\phi)$ and $U(\phi)$ are arbitrary functions (see e.g. 4] and references therein). This form of the action corresponds to the choice of the so-called "Jordan frame", where the scalar field is nonminimally coupled with gravity and all other matter fields obey the equivalence principle. The dynamics of matter fields would be described by an additional term $S_{\text {matt }}$ on the right-hand side, that we set equal to zero because we are interested in BHs in vacuum.

Our numerical evolutions are more easily performed in the so-called Einstein-frame representation, that is related to the Jordan-frame representation by a conformal rescaling of the metric. In the Einstein frame, the scalar field is minimally coupled with the metric tensor and it affects the scalar-matter coupling in the matter action $S_{\text {matt }}$. Working in the Einstein frame is convenient because we focus on pure $\mathrm{BH}$ spacetimes, i.e., we set $S_{\text {matt }}=0$. Since we are interested in the effect of boundary conditions, we shall assume for simplicity (as in [88], and at variance with [85]) that the effect of the scalarfield potential is negligible: $U(\phi)=0$.

\section{A. From Jordan to Einstein and back}

With $U(\phi)=0$, the explicit transformations that recast the previous action in the Einstein frame are [20]

$$
\begin{aligned}
g_{\mu \nu}^{E} & =F(\phi) g_{\mu \nu}, \\
\varphi(\phi) & =\int d \phi\left[\frac{3}{2} \frac{F^{\prime}(\phi)^{2}}{F(\phi)^{2}}+\frac{8 \pi G Z(\phi)}{F(\phi)}\right]^{1 / 2}, \\
A(\varphi) & =F^{-1 / 2}(\phi) .
\end{aligned}
$$

The Einstein-frame action is then

$$
S=\frac{1}{16 \pi G} \int\left[R^{E}-g_{E}^{\mu \nu} \partial_{\mu} \varphi \partial_{\nu} \varphi\right] \sqrt{-g^{E}} d^{4} x,
$$

and it leads to the following equations of motion:

$$
\begin{aligned}
G_{\mu \nu}^{E} & =\partial_{\mu} \varphi \partial_{\nu} \varphi-\frac{1}{2} g_{\mu \nu}^{E} g_{E}^{\rho \sigma} \partial_{\rho} \varphi \partial_{\sigma} \varphi, \\
\square^{E} \varphi & =0,
\end{aligned}
$$

where the label $E$ denotes quantities built out of the Einstein-frame metric $g_{\mu \nu}^{E}$. This will be the starting point of our analysis. It is important to stress that, even though we are formally investigating a minimally coupled theory, our results are in principle applicable to a wide range of scalar-tensor theories. By working in the Einstein frame we can focus on quantities that depend on the intrinsic properties of the binary, rather than quantities that would be measured by gravitational-wave detectors. The latter may be obtained for any specific theory using the transformation from the Einstein frame to the Jordan frame.

\section{B. Gravitational waves in the Einstein and Jordan frames}

In the Jordan frame, we describe perturbations of the metric and of the scalar field as follows:

$$
g_{\mu \nu}=g_{\mu \nu}^{(0)}+h_{\mu \nu}, \quad \phi=\phi^{(0)}+\phi^{(1)} .
$$

In the Einstein frame this corresponds to $g_{\mu \nu}^{E}=g_{\mu \nu}^{(0) E}+$ $h_{\mu \nu}^{E}, \varphi=\varphi^{(1)}$, and from Eqs. $37-(5)$ one gets

$$
\begin{aligned}
& h_{\mu \nu}=F\left(\phi^{(0)}\right)^{-1}\left(h_{\mu \nu}^{E}-g_{\mu \nu}^{(0)} F^{\prime}\left(\phi^{(0)}\right) \phi^{(1)}\right), \\
& \phi^{(1)}=\left[\frac{3}{2} \frac{F^{\prime}\left(\phi^{(0)}\right)^{2}}{F\left(\phi^{(0)}\right)^{2}}+\frac{8 \pi G Z\left(\phi^{(0)}\right)}{F\left(\phi^{(0)}\right)}\right]^{-1 / 2} \varphi^{(1)} .
\end{aligned}
$$

In general, gravitational waves in scalar-tensor theories have 3 degrees of freedom [100, 101. In the Einstein 
frame they correspond to the two transverse-traceless components of the metric perturbation, plus the scalar field. The calculation of these quantities does not present difficulties. The physical degrees of freedom, which should be computed in the Jordan frame, can be read off from Eqs. 10)-(11). An alternative procedure is presented in Ref. 84, which shows the transformation of the corresponding Newman-Penrose quantities, with similar results. In this work we will present results for the scalar field $\varphi$ and for the curvature scalar $\Psi_{4}^{E}$ in the Einstein frame, which is directly related to the two Einstein-frame polarization states $h_{+}^{E}$ and $h_{\times}^{E}$ via

$$
\Psi_{4}^{E}=\ddot{h}_{+}^{E}-i \ddot{h}_{\times}^{E}
$$

where dots denote time derivatives. Quantities in the Jordan frame can be found using Eqs. 10-111, once one specifies the underlying theory. From here onwards, having established the relation between the Einstein and Jordan frames, we shall work exclusively in the Einstein frame, dropping the label "E" from all quantities. Unless specified otherwise, we will use geometrical units and set $G=c=1$. Note however that here $G$ is a bare gravitational constant, and it is different from the quantity measured by a Cavendish experiment.

\section{SCALAR FIELDS IN SINGLE AND BINARY BLACK-HOLE BACKGROUNDS: ANALYTICAL APPROXIMATIONS}

In this Section we introduce approximate analytical solutions that describe single and binary BHs in a scalar field gradient. These solutions will be useful below, either as code checks or for the interpretation of our numerical results.

\section{A. Single black holes: linearized analytical solutions}

Let us assume that the scalar-field gradient is of such low amplitude that the scalar can be treated as a perturbative effect on the spacetime metric. Under this assumption we can neglect terms quadratic in the scalar field, and therefore the field equations reduce to

$$
\begin{aligned}
R_{\mu \nu} & =0, \\
\square \varphi & =0 .
\end{aligned}
$$

We will drop this perturbative approximation in Section IV, where the field equations (7) and (8) will be solved numerically.

Equation 13 is of course identical to Einstein's equations in vacuum. We will consider the Schwarzschild (and later the Kerr) metrics as background $\mathrm{BH}$ solutions, and we will solve the Klein-Gordon equation (14) on these backgrounds. For the reasons explained in the introduction we are interested in numerical evolutions in a scalar-field gradient. Therefore we will consider background scalar-field solutions generated by distant, fixed, infinite homogeneous planes with constant surface scalarcharge density $\sigma$. To our knowledge, Press [102] was the first to study a closely related problem (his setup differs from ours in that he considered a spherical shell of scalar charge as the source of the scalar field). Here we recast some of his results in a form suitable for comparison with our numerical setup.

\section{Spherically symmetric black-hole background}

Let us first consider the Schwarzschild solution in isotropic coordinates:

$$
\begin{aligned}
d s^{2}= & -\frac{\left(1-\frac{M}{2 \hat{r}}\right)^{2}}{\left(1+\frac{M}{2 \hat{r}}\right)^{2}} d t^{2}+\left(1+\frac{M}{2 \hat{r}}\right)^{4}\left(d x^{2}+d y^{2}+d z^{2}\right) \\
= & -\frac{\left(1-\frac{M}{2 \hat{r}}\right)^{2}}{\left(1+\frac{M}{2 \hat{r}}\right)^{2}} d t^{2}+\left(1+\frac{M}{2 \hat{r}}\right)^{4} \\
& \left.\times\left[d \hat{r}^{2}+\hat{r}^{2}\left(d \theta^{2}+\sin ^{2} \theta d \phi^{2}\right)\right)\right]
\end{aligned}
$$

Here $\hat{r}=\sqrt{x^{2}+y^{2}+z^{2}}$ is the isotropic radius, which is related to the areal radius $r$ by $r=\hat{r}\left(1+\frac{M}{2 \hat{r}}\right)^{2}$.

The scalar-field equation (14) on this background admits a simple axisymmetric solution sourced by distant planes of constant scalar-charge density, i.e.:

$$
\begin{aligned}
\varphi_{\mathrm{ext}} & =2 \pi \sigma(r-M) \cos \theta=2 \pi \sigma\left(\hat{r}+\frac{M^{2}}{4 \hat{r}}\right) \cos \theta \\
& =2 \pi \sigma z\left(1+\frac{M^{2}}{4 \hat{r}^{2}}\right)
\end{aligned}
$$

where $z=\hat{r} \cos \theta$ is the direction orthogonal to the charged plane. If no $\mathrm{BH}$ is present, Eq. (16) reduces to the field $\varphi_{\text {ext }}=2 \pi \sigma z$ generated by homogeneously charged infinite planes, with constant gradient $\partial_{z} \varphi_{\text {ext }}=$ $2 \pi \sigma$. For large $|z|$ the constant-gradient behavior applies also to the case where the background is a Schwarzschild $\mathrm{BH}$.

We shall take as initial condition $\varphi_{\text {ini }}=2 \pi \sigma z$ and test our numerical framework by checking that, after a transient, the scalar-field profile settles to the analytical solution $\varphi=\varphi_{\text {ext }}$, up to corrections of second order in the scalar field.

In Appendix $\mathrm{A}$ we derive a solution describing a $\mathrm{BH}$ moving with small constant velocity in a direction orthogonal to the charged planes, and show that it does not emit scalar waves. Indeed, as we will show analytically in Section IIIB and numerically in Section IV, the $\mathrm{BH}$ must have nonvanishing acceleration in order to generate scalar radiation. 

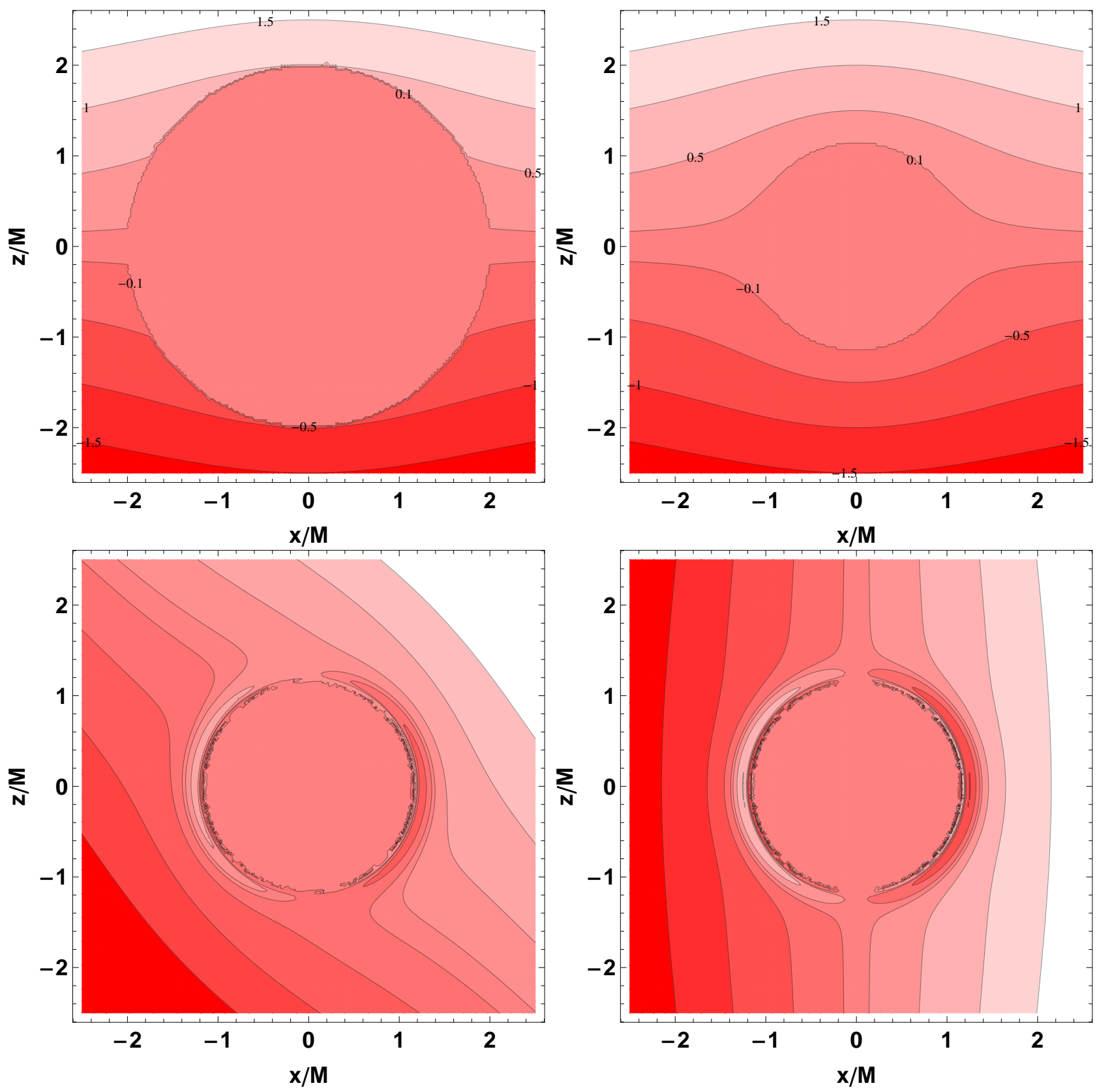

FIG. 1. Contour plots of the field $\varphi_{\text {ext }}$ in the vicinity of a rotating BH, as given by Eq. (17). Top: The infinite charged plane is at an angle $\gamma=0$, and the $\mathrm{BH}$ has dimensionless spin $a=0$ (left) and $a / M=0.99$ (right). The value of $\varphi_{\text {ext }} /(2 \pi \sigma)$ is shown along selected contour lines; the two panels only differ because of the different size of the horizon. Bottom: A BH with $a / M=0.99$ is immersed in a field gradient at angles $\pi / 4$ (left) and $\pi / 2$ (right). All contour plots refer to the plane $y=0$. Selected contour lines correspond to the same values as the top panels.

\section{Rotating black-hole background}

In the case of a rotating $\mathrm{BH}$ with a rotation axis that is not orthogonal to the charged planes, axisymmetry is lost; however, a simple solution can still be found [102]. Let us choose our coordinates so that the $\mathrm{BH}$ spins along the $z$-axis, at an angle $\gamma$ with respect to the direction orthogonal to the charge-carrying planes. Then it can be shown that a solution is

$$
\begin{aligned}
\varphi_{\mathrm{ext}} & =2 \pi \sigma(r-M)\left(\cos \gamma \cos \theta+\sin \gamma \sin \theta \cos \phi f_{a}\right) \\
& =2 \pi \sigma(r-M)\left[\frac{z}{r} \cos \gamma+\frac{x}{r} f_{a} \sin \gamma\right],
\end{aligned}
$$

where $\delta=\sqrt{M^{2}-a^{2}}$,

$$
f_{a}=\frac{1}{r-M} \Re\left[\delta \frac{\Gamma[2-i a / \delta]}{e^{a \frac{\pi}{2 \delta}}} P_{1}^{i a / \delta}\left(\frac{r-M}{\delta}\right)\right],
$$


$\Gamma$ denotes the $\Gamma$-function and $P_{\mu}^{\nu}(z)$ is an associated Legendre function of the first kind. At large distances $f_{a} \rightarrow 1$, and it can be seen that the charged plane generates a uniform gradient in the $x z$-plane. Figure 1 shows contour plots of $\varphi_{\text {ext }}$ in the $y=0$ plane, for different values of $a$ and of the angle $\gamma$. Notice how the field lines are distorted and frame-dragged close to the horizon.

\section{B. Black-hole binaries: analytical approximation for quasi-circular inspirals}

Let us now turn to the more complicated case of a quasi-circular BH binary evolving in an external scalar field with constant gradient. We begin our discussion by addressing the delicate problem of specifying boundary conditions for this system, which will be used to perform the numerical simulations discussed in Sec. IV.

\section{Boundary conditions}

In our numerical setup the boundary conditions are imposed by fictitious faraway charges, which are not modeled in our numerical simulations. These charges are assumed to lie outside the numerical grid, and mimic an external profile due (say) to a galactic scalar-field background. This "large-scale field" acts as a sort of reservoir: when the local field begins falling into the $\mathrm{BH}$, there is an ingoing flux which restores the scalar-field gradient. The presence of this field outside the boundaries of our numerical simulations is implemented through the boundary conditions. At large distances we want to allow for outgoing waves while imposing the existence of the scalar-field gradient, so we require the field to behave in the following way:

$$
\varphi=\varphi_{\mathrm{ext}}+\frac{\Phi(t-r, \theta, \phi)}{r}
$$

where $r$ is the areal radius, the constant gradient corresponds to the external field $\varphi_{\text {ext }}=2 \pi \sigma(r-M) \cos \theta$, and the second term on the right-hand side is the solution of the homogeneous equation $\square \varphi=0$ describing outgoing (approximately spherical) scalar waves. We thus get

$$
\frac{\partial}{\partial r}(r \varphi)+\frac{\partial}{\partial t}(r \varphi)=\frac{\partial}{\partial r}\left(r \varphi_{\mathrm{ext}}\right)
$$

Since the boundary conditions are defined at large distances, $\varphi_{\text {ext }} \simeq 2 \pi \sigma r \cos \theta$, and we can write

$$
\frac{\partial}{\partial r}(r \varphi)+\frac{\partial}{\partial t}(r \varphi)=4 \pi \sigma r \cos \theta
$$

\section{Multipole expansion of the scalar field}

The angular dependence of the scalar field can be described through a multipole expansion of the form

$$
\Phi(t-r, \theta, \phi)=\mathcal{M}+n^{i} \dot{\mathcal{D}}_{i}+\frac{1}{2} n^{i} n^{j} \ddot{\mathcal{Q}}_{i j}+\cdots
$$

where $\Phi$ is the function appearing on the right-hand side of Eq. 19, dots denote derivatives with respect to the retarded null coordinate $u=t-r$ and $\vec{n}=\vec{x} / r=\vec{x} /|\vec{x}|$ is the radial unit vector, which depends only on the angles $(\theta, \phi)$. The calligraphic symbols $\mathcal{M}, \mathcal{D}_{i}$ and $\mathcal{Q}_{i j}$ denote the monopolar, dipolar and quadrupolar components of $\Phi$, respectively.

The full relativistic scalar equation to be solved is $\square \varphi=0$. In order to obtain a solution which describes the physics that we are interested in - namely, a $\mathrm{BH}$ binary in a scalar gradient - it is essential to impose correct boundary conditions, both at null infinity, and in the vicinity of the worldlines of the singularities of the two BHs. The boundary conditions at null infinity have been discussed above; the near-worldline boundary conditions that must be imposed in order to find an approximate solution are more complicated.

In the special case of a comparable-mass $\mathrm{BH}$ binary system with small size-to-separation ratio (or, equivalently, small orbital velocity) the problem of imposing correct near-worldline boundary conditions is substantially simplified when one employs a "point-particle" effective field theory, in which length scales smaller than the Schwarzschild radii are integrated out. In this effective field theory the matter action has the form

$$
S_{\text {matt }}^{\text {pp }}=\sum_{A} \int_{\Gamma_{A}} d s_{A} \mathcal{L}_{A},
$$

where $A$ is an index that runs over the bodies $(A=1,2$ for a binary system), $\Gamma_{A}$ is the worldline of body $A, d s_{A}$ is the proper differential arclength along $\Gamma_{A}$, and $\mathcal{L}_{A}$ is the "effective point-particle Lagrangian" of body A. For a structureless particle of mass $m_{A}$, one has $\mathcal{L}_{A}=-m_{A}$.

The matter action (23) gives rise to sources in the field equations of the effective point-particle theory, and these sources automatically enforce the correct boundary conditions at the worldlines $\Gamma_{A}$ for both $g_{\mu \nu}$ and $\varphi$. For instance, to leading nonrelativistic order, the scalar-field equation has the explicit form

$$
\begin{aligned}
\square_{f} \varphi & =4 \pi \rho_{\varphi} \\
& =4 \pi \sum_{A=1}^{2} Q_{A} \delta^{(3)}\left(\vec{x}-\vec{z}_{A}(t)\right),
\end{aligned}
$$

where $\square_{f}$ is the D'Alembert operator in flat space, $\left(t, \vec{z}_{A}(t)\right)$ is an explicit parametrization of the worldline $\Gamma_{A}$, and $Q_{A}$ are the scalar charges of the BHs.

Moreover, to leading nonrelativistic order, one finds that the multipole moments entering into Eq. (22) are 
given by

$$
\begin{aligned}
& \mathcal{M}=\int d^{3} x \rho_{\varphi}=Q_{1}+Q_{2}, \\
& \overrightarrow{\mathcal{D}}=\int d^{3} x \vec{x} \rho_{\varphi}=Q_{1} \vec{z}_{1}+Q_{2} \vec{z}_{2}
\end{aligned}
$$

and so on.

In general, calculating the scalar charges $Q_{A}$ is a difficult problem. A simplification takes place if we assume that the $\mathrm{BH}$ masses $M_{A}$ have the same order of magnitude $M$, and that

$$
\sigma \ll \sqrt{\frac{a}{M^{3}}} \sim \frac{1}{M v},
$$

where $a$ is the typical orbital separation, and $v$ is the typical orbital velocity. Henceforth, terms of order $(M v \sigma)^{2}$ will be dropped. Then the $\mathrm{BH}$ scalar charges $Q_{A}$ may be found by Jacobson's formula [59], which for Schwarzschild BHs yields

$$
\begin{aligned}
Q_{A}(t) & =4 M_{A}^{2}\left[\frac{\partial \varphi\left(t, \vec{z}_{A}(t)\right)}{\partial t}+\vec{v}_{A}(t) \cdot \vec{\nabla} \varphi\left(t, \vec{z}_{A}(t)\right)\right] \\
& =8 \pi \sigma M_{A}^{2} \vec{v}_{A}(t) \cdot \hat{z}
\end{aligned}
$$

where $\vec{v}_{A}(t)=\dot{\vec{z}}_{A}(t)$ is the velocity of body $A$, and in the second line the full scalar field $\varphi(t, \vec{x})$ has been replaced by the zeroth-order field $\varphi_{\text {ext }}=2 \pi \sigma z$.

Let us specialize to quasi-circular orbits in the $y z$ plane, so that the trajectories take the simple form

$$
\begin{aligned}
\vec{z}_{\mathrm{rel}}(t) & =\vec{z}_{1}(t)-\vec{z}_{2}(t) \\
& =a(t)[\hat{y} \cos \chi(t)+\hat{z} \sin \chi(t)], \\
M \vec{z}_{\mathrm{CM}}(t) & =M_{1} \vec{z}_{1}(t)+M_{2} \vec{z}_{2}(t),
\end{aligned}
$$

where $a(t)$ is the orbital radius, $\chi(t)=\int \omega(t) d t$ is the orbital phase, $\omega(t)$ is the angular frequency of the orbit, and $\vec{z}_{\mathrm{CM}}(t)$ is the center of mass of the binary system. The quantities $\dot{a}, \ddot{\chi}=\dot{\omega}$, and $\vec{a}_{\mathrm{CM}}=\ddot{\vec{z}}_{\mathrm{CM}}$ are all small (of order $\left.1 / c^{5}\right)$, and vanish in the absence of radiation reaction. An explicit expression for their leading-order time evolution may be found by solving the $2.5 \mathrm{PN}$ equations of motion (given in Section 9 of [103]), while dropping conservative corrections. Carrying out this calculation yields

$$
\begin{aligned}
& a(t)=a^{(0)}\left(1-\frac{256 t}{5 \tau_{\mathrm{q}}}\right)^{1 / 4} \simeq a^{(0)}\left(1-\frac{64 t}{5 \tau_{\mathrm{q}}}\right) \\
& \dot{\chi}(t)=\dot{\chi}^{(0)}\left(1-\frac{256 t}{5 \tau_{\mathrm{q}}}\right)^{-3 / 8} \simeq \dot{\chi}^{(0)}\left(1+\frac{96 t}{5 \tau_{\mathrm{q}}}\right)
\end{aligned}
$$

where $a^{(0)}$ and $\dot{\chi}^{(0)}$ are the (constant) radius and angular frequency of the zeroth-order orbit, respectively, and

$$
\tau_{\mathrm{q}}=\left[\frac{M_{1} M_{2}\left(M_{1}+M_{2}\right)}{\left[a^{(0)}\right]^{4}}\right]^{-1}
$$

is the time scale over which the quadrupole tensor radiation shrinks the orbit.
With an explicit description of the orbit in hand, the scalar charges $Q_{1,2}$ and multipole moments $\left.25-26\right)$ may be calculated (see Appendix $\mathrm{B}$ for the explicit expressions). In this way, we find that:

1) monopole radiation is emitted at the orbital frequency:

$$
\begin{aligned}
\mathcal{M} & =\frac{8 \pi \sigma}{M}\left[M\left(M_{1}^{2}+M_{2}^{2}\right)\left(\vec{v}_{\mathrm{CM}} \cdot \hat{z}\right)\right. \\
& \left.+M_{1} M_{2}\left(M_{1}-M_{2}\right)(\dot{a} \sin \chi+a \dot{\chi} \cos \chi)\right],
\end{aligned}
$$

and it vanishes in the equal-mass limit;

2) dipole radiation is emitted at twice the orbital frequency, and more precisely

$$
\dot{\overrightarrow{\mathcal{D}}}=\dot{\overrightarrow{\mathcal{D}}}_{\mathrm{CM}}+\dot{\overrightarrow{\mathcal{D}}}_{\text {rel, DC }}+\dot{\overrightarrow{\mathcal{D}}}_{\text {rel, osc }}
$$

where the "CM" term is emitted at the orbital frequency, the "DC" component is nonoscillatory, and the $\overrightarrow{\mathcal{D}}_{\text {rel, osc }}$ component oscillates at twice the orbital frequency: cf. Eq. B9.

The physical problem addressed here differs from the situation investigated in 88. That study considered time-dependent scalar boundary conditions, rather than a gradient, and it found that monopole radiation is absent, while dipole radiation vanishes in the equal-mass limit. One may expect dipole scalar radiation to be emitted at the orbital frequency, rather than twice the orbital frequency. The reason why this expectation is erroneous in our case is that we have a background field with a gradient directed along the orbital plane, which combines with the oscillatory component sourced by the orbital motion.

A simple toy model can provide us with a complementary and perhaps more intuitive way to justify the expectation that dipole radiation must be emitted at twice the orbital frequency. Let us consider a rotating source with frequency $\Omega$ on a scalar-field background $\varphi_{\text {ext }}=2 \pi \sigma z=2 \pi \sigma r \sin \theta \sin \phi$ [in our "rotated" polar coordinates, see Eqs. (74) below]. The source will produce a modulation in the background field of the form

$$
\varphi=\varphi_{\mathrm{ext}}[1+f(\phi-\Omega t)]
$$

Expanding in circular harmonics, $f(\phi-\Omega t)=$ $\sum_{m} f_{m} e^{\mathrm{i} m(\phi-\Omega t)}$ and

$$
\varphi=2 \pi \sigma r \sin \theta \sin \phi\left(1+\sum_{m} f_{m} e^{\mathrm{i} m(\phi-\Omega t)}\right),
$$

which implies that the multipolar components of the field will have the following dependence:

$$
\varphi_{l m} \sim\left(e^{-\mathrm{i}(m+1) \Omega t}+e^{-\mathrm{i}(m-1) \Omega t}\right)+\text { constant } .
$$


Therefore the $m=0$ contribution should oscillate with frequency $\Omega$, the $m=1$ contribution with frequency $2 \Omega$, the $m=2$ contribution with frequencies $3 \Omega$ and $\Omega$, and so on. As we will show below, this behavior is consistent with our numerical simulations.

\section{NUMERICAL IMPLEMENTATION}

Our numerical implementation of scalar-tensor theory closely parallels [104, but borrowing notation and conventions from [105]. The physical system studied in 105] was quite different, since that paper considered higherdimensional Einstein gravity in vacuum. However the equations can be cast as a system involving a scalar field coupled to gravity via dimensional reduction, so they are formally similar to the system considered here, as we show below.

\section{A. $3+1$ decomposition}

As a preliminary step for our numerical implementation, we perform a $3+1$ decomposition of the spacetime (see [106] and references therein). Let us consider a slicing of the spacetime in a set of three-dimensional surfaces $\Sigma$. Introducing the normal $n_{\mu}$ to the surface $\Sigma$ and the projector

$$
\gamma_{\mu \nu}=g_{\mu \nu}+n_{\mu} n_{\nu}
$$

we write the four-dimensional metric in the form $(\mu, \nu=$ $0, \ldots, 3 ; i, j=1,2,3)$ :

$$
\begin{aligned}
d s^{2} & =g_{\mu \nu} d x^{\mu} d x^{\nu} \\
& =-\alpha^{2} d t^{2}+\gamma_{i j}\left(d x^{i}+\beta^{i} d t\right)\left(d x^{j}+\beta^{j} d t\right)
\end{aligned}
$$

where $\alpha, \beta^{i}$ are the lapse and the shift, respectively, and

$$
\partial_{t}=\alpha n+\beta
$$

We shall denote by $D_{i}$ the covariant derivative on $\Sigma$, i.e., the covariant derivative with respect to the threedimensional metric $\gamma_{i j}$.

The Lie derivative with respect to $n^{\mu}$, then, is $\mathcal{L}_{n}=$ $\left(\partial_{t}-\mathcal{L}_{\beta}\right) / \alpha$. Defining the extrinsic curvature

$$
K_{i j} \equiv-\frac{1}{2} \mathcal{L}_{n} \gamma_{i j}
$$

we have the following evolution equations for the (threedimensional) metric:

$$
\left(\partial_{t}-\mathcal{L}_{\beta}\right) \gamma_{i j}=-2 \alpha K_{i j}
$$

Furthermore, we define the scalar curvature $K_{\varphi}$ as

$$
K_{\varphi}=-\frac{1}{2} \mathcal{L}_{n} \varphi
$$

so that

$$
\left(\partial_{t}-\mathcal{L}_{\beta}\right) \varphi=-2 \alpha K_{\varphi}
$$

Comparing with the definitions of the variables $Q_{i}$ and $\Pi$ in [104, we find that

$$
\begin{aligned}
Q_{i} & =\frac{D_{i} \varphi}{\sqrt{8 \pi G}}=\frac{\gamma_{i \mu} \partial^{\mu} \varphi}{\sqrt{8 \pi G}}, \\
\Pi & =\frac{\mathcal{L}_{n} \varphi}{\sqrt{8 \pi G}} .
\end{aligned}
$$

Therefore,

$$
K_{\varphi}=-\frac{1}{2} \sqrt{8 \pi G} \Pi .
$$

The constraint equations (2.15) and (2.16) of [104] read

$$
{ }^{(3)} R+K^{2}-K_{i j} K^{i j}=\frac{\Pi^{2}+Q^{2}}{f}=4 K_{\varphi}^{2}+D_{i} \varphi D^{i} \varphi
$$

$$
D_{l} K_{i}^{l}-D_{i} K=-\frac{\Pi Q_{i}}{f}=2 K_{\varphi} D_{i} \varphi .
$$

The evolution equation (2.17) of [104] can be written as

$$
\begin{aligned}
& \left(\partial_{t}-\mathcal{L}_{\beta}\right) K_{i j}=\left(\partial_{t}-\mathcal{L}_{\beta}\right) \gamma_{i k} K_{j}^{k} \\
& =-D_{i} D_{j} \alpha+\alpha\left({ }^{(3)} R_{i j}+K K_{i j}-D_{i} \varphi D_{j} \varphi-2 K_{i k} K_{j}^{k}\right)
\end{aligned}
$$

Note that since $\gamma_{i}^{\mu} \gamma_{j}^{\nu} R_{\mu \nu}=D_{i} \varphi D_{j} \varphi$, this expression coincides with Eq. (2.23) of [105]. Taking the trace of Eq. 50 we have

$$
\partial_{t} K-\beta^{l} \partial_{l} K+D^{i} D_{i} \alpha-\alpha\left({ }^{(3)} R+K^{2}-D^{i} \varphi D_{i} \varphi\right)
$$

and using Eq. 48 we find

$$
\left(\partial_{t}-\mathcal{L}_{\beta}\right) K=-D^{i} D_{i} \alpha+\alpha K_{i j} K^{i j}+4 \alpha K_{\varphi}^{2},
$$

that should be compared to Eq. (2.19) of 104. The evolution equation (2.18) of [104] can be written as

$$
\begin{aligned}
& \frac{1}{\alpha}\left(\partial_{t}-\mathcal{L}_{\beta}\right) K_{\varphi}=-\frac{1}{2 \sqrt{8 \pi G}} \mathcal{L}_{n} \Pi \\
& =K_{\varphi} K-\frac{1}{2 \alpha} D^{i} \varphi D_{i} \alpha-\frac{1}{2} D_{i} D^{i} \varphi .
\end{aligned}
$$

All terms of this expression appear, with the same coefficients, in Eq. (2.37) of [105. The additional terms in that equation which are not present here are due to the more complicated dynamics of the scalar field arising from dimensional reduction.

It can be useful to write the equations also in terms of the stress-energy tensor, which enables us to compare the scalar-field terms with those in [106]. From (7) we have

$$
8 \pi G T_{\mu \nu}=\partial_{\mu} \varphi \partial_{\nu} \varphi-\frac{1}{2} g_{\mu \nu} \partial_{\alpha} \varphi \partial^{\alpha} \varphi
$$


Since $n^{\mu} \partial_{\mu} \varphi=-2 K_{\varphi}, \gamma^{\mu \nu} \partial_{\mu} \varphi=D^{\nu} \varphi, g_{\mu \nu}=\gamma_{\mu \nu}-$ $n_{\mu} n_{\nu}$, defining, as on page 87 of [106]

$$
\begin{aligned}
\rho & =n^{\mu} n^{\nu} T_{\mu \nu}, \\
j^{\alpha} & =-\gamma^{\alpha \mu} n^{\nu} T_{\mu \nu},
\end{aligned}
$$

we get

$$
\begin{aligned}
8 \pi G \rho & =n^{\mu} n^{\nu} \partial_{\mu} \varphi \partial_{\nu} \varphi-\frac{1}{2} g_{\mu \nu} \partial_{\alpha} \varphi \partial^{\alpha} \varphi \\
& =2 K_{\varphi}^{2}+\frac{1}{2} D_{i} \varphi D^{i} \varphi \\
8 \pi G j^{i} & =-8 \pi G \gamma^{i \mu} n^{\nu} T_{\mu \nu} \\
& =-D^{i} \varphi n^{\mu} \partial_{\mu} \varphi=2 K_{\varphi} D^{i} \varphi
\end{aligned}
$$

where we have used the fact that $\gamma^{00}=0$ (see [106]), thus $\gamma^{\mu \nu} D_{\mu} \varphi D_{\nu} \varphi=\gamma^{i j} D_{i} \varphi D_{j} \varphi$. Therefore, Eqs. (2.4.6) and (2.4.9) coincide with our constraint equations (48) and (49). Furthermore, we can compute the quantity (cf. page 89 of [106])

$$
S^{\mu \nu}=\gamma^{\mu \alpha} \gamma^{\nu \beta} T_{\alpha \beta} .
$$

We have

$$
8 \pi G S^{\mu \nu}=D^{\mu} \varphi D^{\nu} \varphi-\frac{1}{2} \gamma^{\mu \nu} D^{i} \varphi D_{i} \varphi+2 \gamma^{\mu \nu} K_{\varphi}^{2},
$$

and the trace of this equation ( since $\gamma_{\mu}^{\mu}=3$ ) yields

$$
8 \pi G S=-\frac{1}{2} D^{i} \varphi D_{i} \varphi+6 K_{\varphi}^{2} .
$$

Then, $8 \pi G(S-\rho)=4 K_{\varphi}^{2}-D_{i} \varphi D^{i} \varphi$, and

$$
4 \pi G\left[(S-\rho) \gamma_{i j}-2 S_{i j}\right]=-D_{i} \varphi D_{j} \varphi,
$$

therefore Eq. (2.5.6) of [106 coincides with our Eq. 50.

\section{B. Baumgarte-Shapiro-Shibata-Nakamura formalism}

Our evolution equations use the Baumgarte-ShapiroShibata-Nakamura (BSSN) formalism, in which the dynamical variables are $\left\{\chi, \gamma_{i j}, \tilde{A}_{i j}, K, \tilde{\Gamma}^{i}\right\}$, defined as follows:

$$
\begin{aligned}
& \gamma_{i j}=\chi^{-1} \tilde{\gamma}_{i j} \quad\left(\text { with } \gamma^{i j}=\chi \tilde{\gamma}^{i j}\right) \\
& \chi=\left(\operatorname{det} \gamma_{i j}\right)^{-1 / 3} \\
& \tilde{A}_{i j}=\chi\left(K_{i j}-\frac{1}{3} \gamma_{i j} K\right) \\
& \Gamma^{k}=\gamma^{i j} \Gamma_{i j}^{k}=\chi \tilde{\Gamma}^{k}+\frac{1}{2} \tilde{\gamma}^{k j} \partial_{i} \chi .
\end{aligned}
$$

Alternative notations replace our variable $\chi$ by a variable $\psi$ defined as $\chi^{-1}=\psi^{4}$. Here we will use $\chi$ as our dynamical quantity.

The Einstein equations in the BSSN formulation in the presence of a scalar field, which appears through the quantities $\rho, j^{i}, S_{i j}$, are implemented in an extended version of the CACTUS [107] based LEAN code [108, 109 in the form given in Eqs. (C1)-(C10) of Appendix C. Mesh refinement for our simulations is provided by CARPET [110, horizon diagnostics by AHFInDERDIRECT [111, 112] and BH binary initial data satisfying Eq. 13. by a spectral solver [113] provided through CACTUS as the TwoPunctures thorn.

\section{Initial data}

Our initial data sets consist of either a single $\mathrm{BH}$ or a BH binary evolving in a background scalar field with a nonvanishing gradient. The background scalar field is generated by distant sources, which are kept fixed in our time evolutions. As a simple way to enforce this scenario, imagine that the background scalar field is generated by infinite homogeneous charged planes with surface density $\sigma$. As we saw in Section III, if a BH is present in the spacetime and the scalar field is small enough to be treated in the linear approximation the metric is unaffected, but the equilibrium solution for the scalar field changes. This setup is quite similar in spirit to the one adopted by Palenzuela and collaborators [114, 115], the main difference being that they dealt with electromagnetic fields, and that their external magnetic field is generated by a current loop far away from the system.

Under these assumptions, the solution $\varphi_{\text {ext }}$ we found in Eq. (16) should be a good approximation to the initial data describing a single, nonrotating $\mathrm{BH}$ in a scalar-field gradient. Except for regions close to the $\mathrm{BH}$ singularity, the term $M^{2} / 4 \hat{r}^{2}$ in parentheses in Eq. (16) can furthermore be neglected. Therefore we initialize the scalar field using the simplified expression $\varphi_{\text {ext }}=2 \pi \sigma z$. Our numerical simulations of single BHs show indeed that the initial data (16) and its approximate version $2 \pi \sigma z$ yield (after a brief transient, which we exclude from our analysis below) virtually identical evolutions of the scalar field and of the spacetime metric.

\section{Upper bounds on the field gradient from the threshold of black-hole formation}

Our setup consists of a constant scalar-field gradient at large distances. Because the energy density in any classical field theory is proportional to the square of the field gradient, one expects a roughly constant energy density $\rho \sim \sigma^{2}$. The total mass $M$ in a region of linear dimension $R$ then scales like $\sim \rho R^{3}$, and therefore $M / R \sim \rho R^{2} \sim \sigma^{2} R^{2}$. Therefore we expect that the initial data will contain a horizon for $\sigma \gtrsim R^{-1}$. This condition imposes a nontrivial constraint on the size of our numerical grid. Here we provide a more formal argument supporting this conclusion.

We focus on conformally flat backgrounds with $\tilde{A}^{j}{ }_{i}=$ $0, \Pi=0$. Then the momentum constraint is identically 
satisfied, while the Hamiltonian constraint yields

$$
0=\triangle \psi+\frac{1}{8} \psi \eta^{i j} \partial_{i} \varphi \partial_{j} \varphi
$$

where $\eta^{i j}$ is the Minkowski metric. A scalar field with constant gradient is such that $\varphi=2 \pi \sigma z$, and therefore we get the equation

$$
\triangle \psi=-\frac{\pi^{2} \sigma^{2}}{2} \psi
$$

Imposing regularity at $r=0$, the solution of this equation is

$$
\psi=\frac{A \sin (\pi \sigma r / \sqrt{2})}{r} .
$$

An apparent horizon exists for $\left(\psi^{4} r^{2}\right)^{\prime}=0$. This condition is equivalent to finding the roots of

$$
x \cot x=1 / 2, \quad \text { with } \quad x \equiv \frac{\pi \sigma r}{\sqrt{2}} .
$$

The smallest root of this equation is at $x \simeq 1.16556$, i.e.

$$
\sigma \simeq 1.16556 \frac{\sqrt{2}}{\pi r} \simeq \frac{0.52469}{r}
$$

in good agreement with the previous back-of-theenvelope estimate.

Our boundary conditions are enforced at distance $r / M=160$ and 384, respectively, for single and binary $\mathrm{BH}$ simulations. This provides a formal upper bound of $M \sigma \approx 5 \times 10^{-3}$ on the magnitude of the gradients that we can simulate. However, as we will see below, even smaller gradients $M \sigma \approx 10^{-4}$ can generate exponentially growing instabilities (indicating collapse) in our numerical evolutions.

\section{NUMERICAL RESULTS}

In this Section we first discuss our results for single $\mathrm{BH}$ evolutions, verifying that for small values of the gradient $M \sigma$ they are in good agreement with the analytical predictions of Section III A. Then we study gravitational and scalar radiation from $\mathrm{BH}$ binaries in a scalar-field gradient.

\section{A. Single black-hole evolutions}

In order to compare our numerical results with the analytical predictions of Section III it is important to minimize coordinate effects. Let us denote by $\tilde{r}$ the radial coordinate used in our numerical simulation, which coincides with the isotropic coordinate at $t=0$, i.e. $\tilde{r}(t=0)=\hat{r}$. This need not be true at later times, since the gauge can dynamically change during the evolution. In order to monitor the scalar field as a function of time

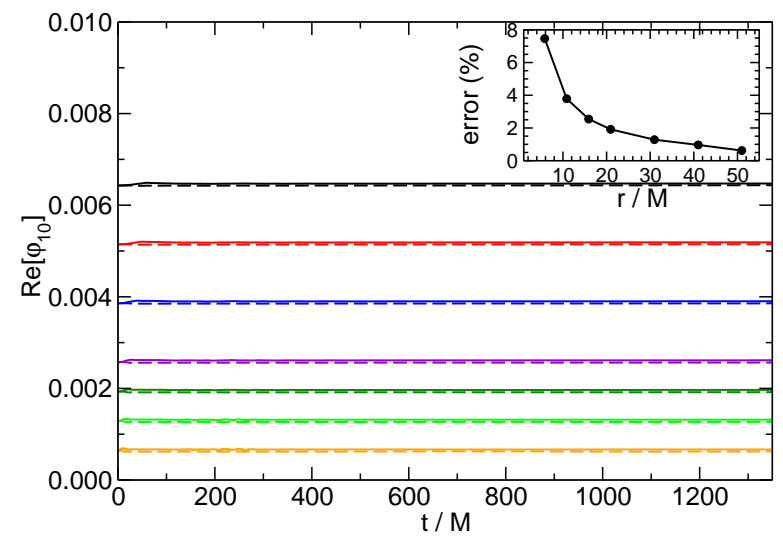

FIG. 2. Real part of the scalar dipole mode $\varphi_{10}$ (the imaginary part vanishes) for $M \sigma=10^{-5}$ and extraction radii (from top to bottom) $\tilde{r} / M=50,40,30,20,15,10$ and 5, compared to the predictions of Eq. 76 . Solid lines refer to the numerical evolution; dashed lines refer to the analytical solution evaluated at time-dependent areal radii $r$, which are computed dynamically during the evolution. The inset shows the percentage discrepancy between the numerical and analytical prediction as a function of extraction radius.

and check that it eventually settles to a configuration close to the analytical solution $\varphi_{\text {ext }}$ of Eq. 16 , we need to perform integrations over spheres at given values of $\tilde{r}$. Furthermore, we compute the areal radii $r$ at these locations by performing spherical integrations of the metric components, as discussed in Ref. [116.

The spherical-harmonic expansion ${ }^{2}$ of the scalar field reads

$$
\varphi(t, r, \theta, \phi)=\sum_{l m} \varphi_{l m}(t, r) Y_{l m}(\theta, \phi)
$$

where

$$
\varphi_{l m}(t, r)=\int d \Omega \varphi(t, r, \theta, \phi) Y_{l m}^{*}(\theta, \phi) .
$$

Since the binary moves in the $y z$-plane, we use noncanonical rotated coordinates

$$
\begin{aligned}
& x=r \cos \theta, \\
& y=r \sin \theta \cos \phi, \\
& z=r \sin \theta \sin \phi .
\end{aligned}
$$

2 This multipole expansion can be simply related to that introduced in Eq. 22. For example, we have:

$$
\dot{\overrightarrow{\mathcal{D}}}=\frac{r}{2} \sqrt{\frac{3}{\pi}}\left[\varphi_{10} \hat{x}-\frac{\varphi_{11}-\varphi_{1-1}}{\sqrt{2}} \hat{y}-i \frac{\varphi_{11}+\varphi_{1-1}}{\sqrt{2}} \hat{z}\right]_{1 / r},
$$

where the subscript $1 / r$ denotes that only the $1 / r$ term should be kept. 


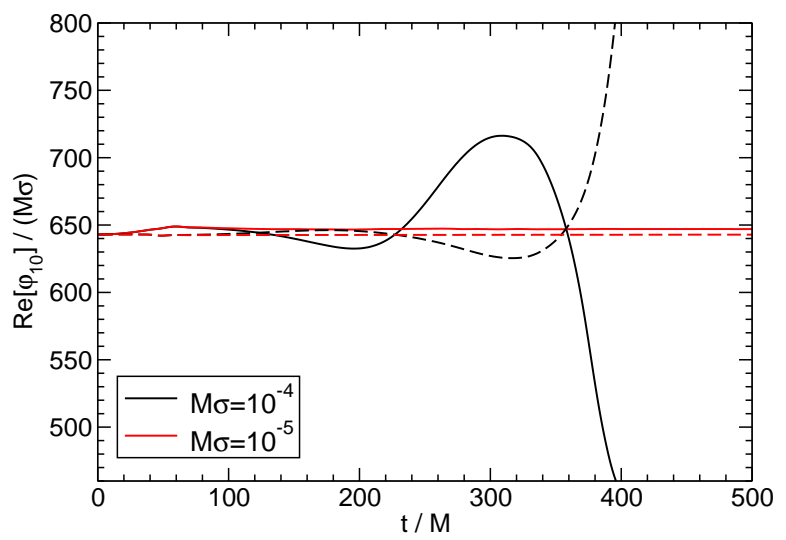

FIG. 3. Real part of the scalar dipole mode $\varphi_{10}$ (rescaled by $M \sigma$ ) at the largest extraction radius $\tilde{r} / M=50$ for $M \sigma=10^{-4}$ and $M \sigma=10^{-5}$, compared to the predictions of Eq. 76. Solid lines refer to the numerical evolution; dashed lines refer to the analytical solution evaluated at timedependent areal radii $r$, which are computed dynamically during the evolution. The evolution does not settle to the analytical solution for $M \sigma=10^{-4}$ : there is an exponentially growing mode. This also shows up as an exponential growth of the subleading multipoles, as can be seen in Fig. 4.

For small scalar-field gradients, the back-reaction on the metric is very small and we expect to recover the stationary solution $\varphi_{\text {ext }}$ found in Section IIIA i.e., we expect that after an initial transient

$$
\varphi(t \rightarrow \infty, r, \theta, \phi)=\varphi_{\mathrm{ext}}(r, \theta)=\varphi_{0}+2 \pi \sigma(r-M) \cos \theta .
$$

where $\varphi_{0}$ is a constant. Because $\left|Y_{10}\right|=\sqrt{3 /(4 \pi)} \cos \theta$, the dominant nonvanishing component $\varphi_{l m}$ at late times (up to quadratic corrections in the field) should be given by

$$
\varphi_{10}=\sqrt{\frac{4 \pi}{3}} 2 \pi \sigma(r-M) .
$$

In this expression the areal radius $r$ is effectively timedependent, and it is computed dynamically during the evolution as described above.

The numerical and analytical predictions (solid and dashed lines, respectively) are compared in Figs. 2 and 3. Let us focus first on Fig. 2, which refers to $M \sigma=$ $10^{-5}$. In this case the numerically extracted dipole mode asymptotes quickly to the analytical prediction. The inset shows the percentage difference between the analytical and numerical value of the scalar field at late times as a function of the extraction radius: the agreement is remarkable at large radii but it gets progressively worse as we get closer to the $\mathrm{BH}$, most likely because of gauge effects (we can exclude that the deviations are due to nonlinear effects, because these would scale with $\sigma^{2}$, whereas the disagreement seems to be independent of $\sigma$ ). To summarize, the analytical and numerical predictions agree within a few percent for gradients $M \sigma \leq 10^{-5}$.

The situation changes significantly for larger values of $M \sigma$. In Fig. 3 we overplot the numerical and analytical components for $M \sigma=10^{-5}$ and for a larger gradient, $M \sigma=10^{-4}$. As expected, when rescaled by their respective gradients the dipolar components are essentially the same, and they also converge to the value predicted by the analytical solution. However for $M \sigma=10^{-4}$ this convergence can only be seen at intermediate times, whereas at late times the mode develops an instability: the figure shows that the roughly constant "baseline" value of the field seems to be superimposed to an exponentially growing oscillation that develops on timescales $t \sim 10^{2} \mathrm{M}$, both when we evaluate the field numerically (continuous black line) and when we use the areal radii to compute the analytical solution (dashed black line).

The existence of this instability is confirmed by Fig. 4 . where we look at higher multipoles of the field on a logarithmic scale for $M \sigma=10^{-5}$ (left panel) and for $M \sigma=10^{-4}$ (right panel). The right panel of this plot shows that for the larger gradient $M \sigma=10^{-4}$ an exponentially growing instability with growth time $\sim 10^{2} \mathrm{M}$ is present also in the subdominant $l=0, l=2$ and $l=3$ multipoles. The left panel illustrates that when $M \sigma=10^{-5}$ the instability (if it is present at all) develops on much longer timescales $t \gtrsim 10^{3} \mathrm{M}$, so it does not affect our numerical simulations. Notice that earlyintermediate time results are physically consistent: for both values of the gradient the scalar-field distribution is dominated by the dipolar component, and it is in good agreement with analytical predictions.

In summary, single-BH simulations in a scalar-field gradient show that our numerical evolutions are stable and reliable as long as the gradient is not too large. This conclusion is compatible with the arguments presented in Section IVD above.

\section{B. Binary black-hole evolutions: scalar and gravitational radiation}

In this Section we discuss our numerical evolutions of $\mathrm{BH}$ binaries in a scalar-field gradient. In this initial study we focus on evolutions of nonspinning, unequal-mass binaries with a gradient $M \sigma=2 \times 10^{-7}$ and mass ratio $q=3$, because unequal-mass binaries display two interesting features which would be absent by construction in the equal-mass case: center-of-mass recoil [see Eqs. (B1)[B3)] and monopole scalar radiation [see Eq. (B4)].

Gravitational waveforms, as characterized by the Newman-Penrose scalar $\Psi_{4}$, are shown in Fig. 5. For such low values of the scalar-field gradient, the impact on gravitational radiation emission is hardly noticeable.

The emission of scalar radiation is much more interesting. The scalar field acquires a nontrivial profile due to the dynamics of the orbiting $\mathrm{BH}$ binary. The scalar 

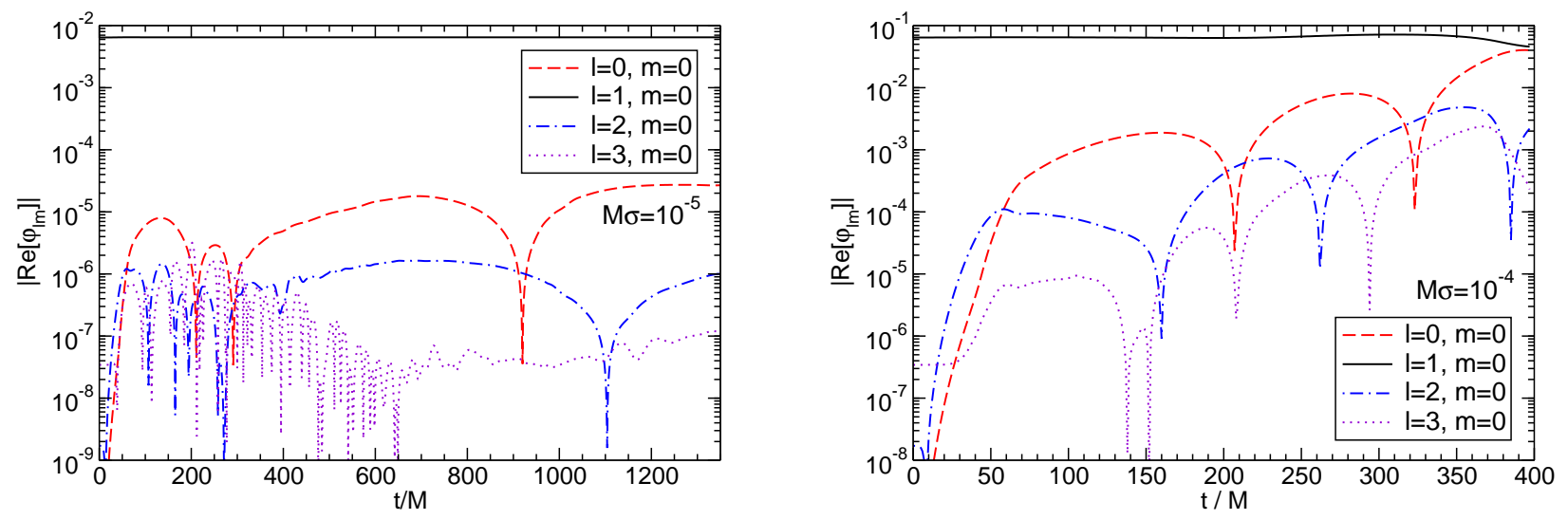

FIG. 4. Absolute value of the real part of the scalar multipoles $\left|\operatorname{Re}\left(\varphi_{l 0}\right)\right|$ evaluated at the largest extraction radius $\tilde{r}=50 M$ for different values of $l$ and two values of the scalar-field gradient, $M \sigma=10^{-5}$ and $M \sigma=10^{-4}$ (left and right panel, respectively).
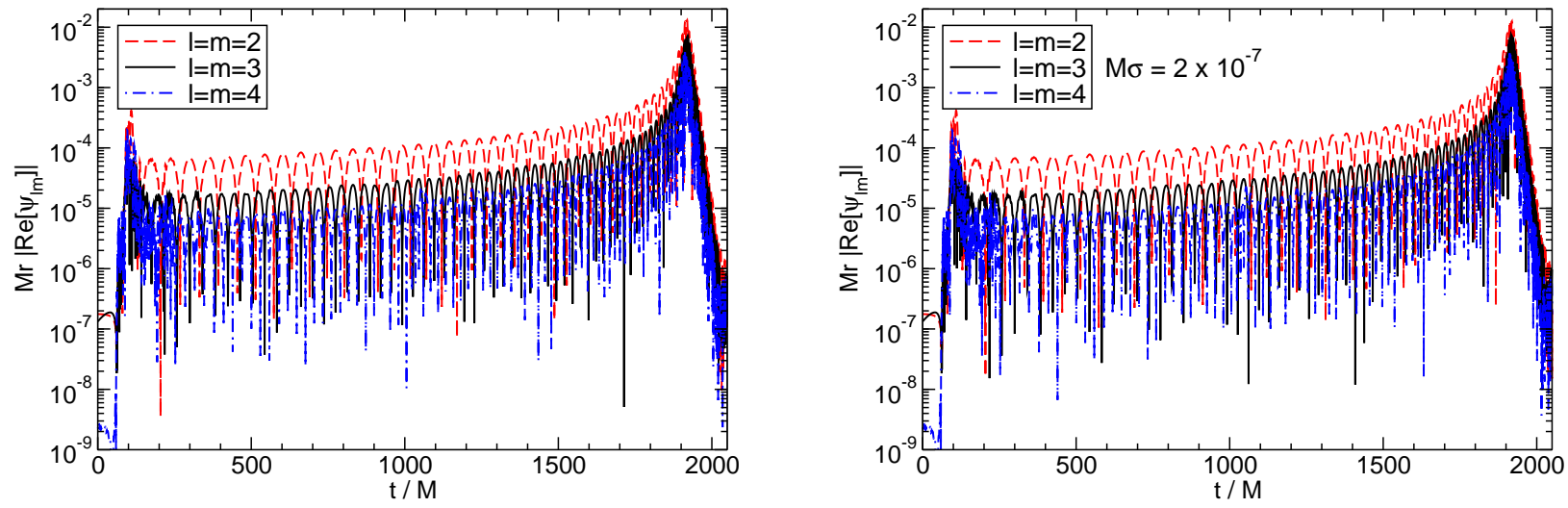

FIG. 5. Numerical results for a BH binary inspiralling in a scalar field gradient, with the orbital angular momentum perpendicular to the gradient. We show the spin-weighted spheroidal harmonic components of the Weyl scalar $\Psi_{4},\left|\operatorname{Re}\left(\psi_{l m}\right)\right|$, extracted at $r=56 M$ for $l=m$ (the imaginary parts are identical, modulo a phase shift). Left: $M \sigma=0$, right: $M \sigma=2 \times 10^{-7}$.

radiation has a crucial dependence on the binary setup, and more specifically on the angle between the orbital angular momentum of the binary and the direction of the scalar-field gradient. If this angle is zero, then effectively the individual BHs do not traverse any field gradient, and the scalar profile is expected to be trivial. Our numerical results confirm this expectation: the output of these simulations is indistinguishable from vacuum evolutions in pure general relativity [109, 117.

On the other hand, the induced scalar radiation should be maximized when the orbital angular momentum is perpendicular to the field gradient, so we now focus on this case. Our results are summarized in Figs. 5 and 6 .

Because the binary evolves on the background of a dipolar scalar-field profile, this constant "background" value of the scalar shows up as a large imaginary component $t^{3}$ of the $l=|m|=1$ scalar-field modes, which is apparent in the left panel of Fig. 6] (in fact, we had to rescale the imaginary component by a factor $10^{-3}$ in

\footnotetext{
${ }^{3}$ In both the single-BH solution 17 with $\gamma=\pi / 2$ and in the numerical solution discussed here, the polar axis (in terms of which the polar angles, and then the harmonic decomposition, are defined) is orthogonal to the gradient. However the ordering of the axes is different in the two cases. This explains why the imaginary part $\operatorname{Im}\left(\varphi_{11}\right)$ of the numerical solution corresponds to the real part $\operatorname{Re}\left(\varphi_{11}\right)$ of the analytical solution.
} 

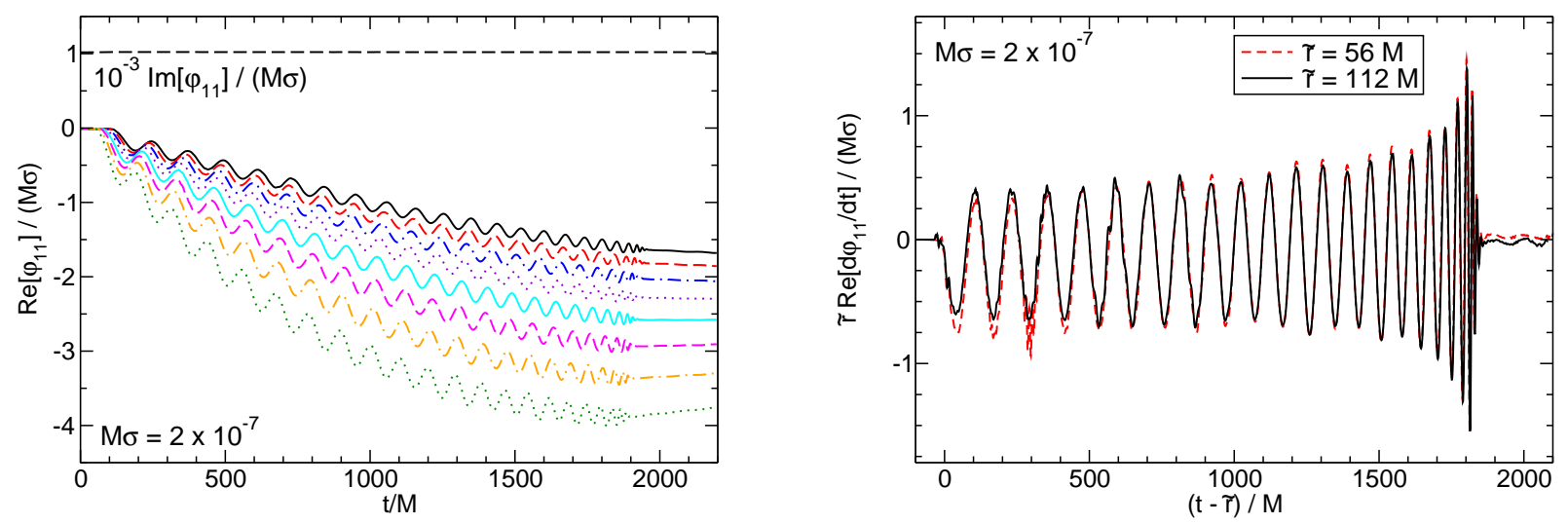

FIG. 6. Numerical results for a BH binary inspiralling in a scalar field gradient, with the orbital angular momentum perpendicular to the gradient. Left: dependence of the various components of the scalar radiation $\operatorname{Re}\left(\varphi_{11}\right) /(M \sigma)$ on the extraction radius (top to bottom: $112 M$ to $56 M$ in equidistant steps). The dashed line corresponds instead to $10^{-3} \operatorname{Im}\left(\varphi_{11}\right) /(M \sigma)$ at the largest extraction radius. Right: time-derivative of the scalar field at the largest and smallest extraction radii, rescaled by radius and shifted in time. Notice how the two waveforms show a clean and typical merger pattern, and that they overlap showing that the field scales to good approximation as $1 / \tilde{r}$.

order to show this on this plot).

The real part of $\varphi_{11}$ displays interesting dynamics (the imaginary component also has similar dynamics, but this is partially masked by the large background dipolar field, so the analysis of the real part turns out to be numerically "cleaner.") At any extraction radius $\operatorname{Re}\left(\varphi_{11}\right)$ is initially zero, as the binary is simply traversing a constant scalar field. As the binary evolves, we expect to see scalar radiation crossing the extraction surface and producing a nonvanishing scalar profile. This is indeed observed in Fig. 6, where we show that $\operatorname{Re}\left(\varphi_{11}\right)$ has a "wavy" pattern at any fixed extraction radius. The scalar-wave nature of this pattern is well illustrated by the right panel of Fig. 6. There we take the time derivative of $\operatorname{Re}\left(\varphi_{11}\right)$ at the largest and smallest extraction radii, scaling the amplitude of the signal by the ratio of the extraction radii (as expected for a wave scaling like $1 / r$ ), and we observe that the signal is indeed dipole scalar radiation emitted at twice the orbital frequency of the binary, consistent with the predictions of Sec. III B 2. Furthermore, in Eq. (B15) of Appendix B we show that the amplitude of the $\varphi_{11}$ mode is consistent in order of magnitude with analytical predictions. We also observe a monopole component $\varphi_{00}$ whose amplitude is comparable to the amplitude of $\varphi_{11}$, consistently with analytical predictions.

To understand the merger signal, when the two BHs collide and relax to a final nearly stationary state, it is useful to remember that, in vacuum, the merger of a $\mathrm{BH}$ binary with mass ratio $q=3$ produces a Kerr $\mathrm{BH}$ with spin $a / M \sim 0.543$ [117. Thus the lowest ringdown frequencies are expected to be, from perturbative calculations, $M \omega=0.351-0.0936 i, 0.476-0.0849 i$ for a $l=m=1$ scalar field, $l=m=2$ gravitational mode [118, 119]. We find that $\Psi_{4}$ indeed rings down with $M \omega \sim 0.48-0.081 i$, in good agreement with perturbative calculations. An analysis of $\dot{\varphi}_{11}$ yields a ringdown frequency $M \omega \sim 0.36-0.070 i$ (with errors $\lesssim 10 \%$ ), which is roughly consistent with perturbative calculations of scalar $(s=0)$ perturbations of Kerr BHs [119]. Our simulations also show that the ringdown phase, and indeed the entire scalar signal, scales with $\sigma$. We conclude that our results indeed represent linear effects, as opposed to nonlinear mode couplings.

Although not completely obvious, there is a small DC component in Fig. 6 (right panel), which we estimate to be

$$
\frac{\left|\dot{\varphi}_{11}^{D C}\right|}{\left|\dot{\varphi}_{11}^{\text {peak }}\right|} \sim 0.2,
$$

at early times, where $\left|\dot{\varphi}_{11}^{\text {peak }}\right|$ is the absolute value of the waveform at a local peak (maximum or minimum). This numerical estimate can be compared to the analytical prediction, Eqs. (B11)-(B12). We start by estimating the angular frequency $\dot{\chi}$ through the waveform frequency, and we find $M \dot{\chi} \sim 0.025$. Using Kepler's law, one can estimate the orbital separation, and these two ingredients together with relations (32) allow us to estimate the relevant ratio of the time derivatives of expressions (B11)-(B12). We find a ratio which is smaller by almost one order of magnitude. This discrepancy can probably be explained by numerical uncertainties and strong-field nonlinear effects.

It is apparent from Fig. 6 (left panel) that the $\operatorname{Re}\left(\varphi_{11}\right)$ modes display a "drift": after all the dynamics has died 
away, the field does not return to zero. Our data implies that at late times $\operatorname{Re}\left(\varphi_{11}\right) \sim-2.7 \times 10^{-4} r^{-1.24}$ for $M \sigma=$ $2 \times 10^{-7}$. The most natural interpretation of this drift is related to the DC component, Eq. (B5), which predicts a linear growth in time - roughly the same dependence that can be seen in Fig. 6. One should also bear in mind that the analytical result is a slow-motion expansion, whereas the numerical results cover only the highly dynamical, nonlinear merger signal; some deviation from a perfectly linear dependence is therefore expected.

There are other possible contributions to such a drift. A second possible contribution is due to the nonvanishing of $\operatorname{Im}\left(\varphi_{11}\right)$ for the analytical solution (17) with $\gamma=\pi / 2$ (recall that in both the single-BH solution (17) and in the numerical solution discussed here, the polar axis is orthogonal to the gradient, but the ordering of the axes is different, which explains why real and imaginary parts of the modes are swapped). However, the $1 / r$ piece of that solution is orders of magnitude smaller than the amplitude of the drift we observe numerically, and therefore unlikely to explain our observations.

Another possible contribution comes from gravitational recoil. We are simulating an unequal mass binary, which acquires a kick from the origin of our coordinate axis as a result of the merger. The kick introduces "spurious" multipolar components with respect to a frame which is not comoving with the final BH. However, an order-of-magnitude estimate shows that this effect is unlikely to explain the observed drift. In order of magnitude, the kick contribution to $\varphi$ can be estimated by looking at the terms in Eq. B10, say $\dot{\overrightarrow{\mathcal{D}}}_{\text {recoil }}$, that are proportional to $v_{\mathrm{CM}}$ : this yields

$$
\left|\frac{\dot{\overrightarrow{\mathcal{D}}}_{\text {recoil }}}{\tilde{r}}\right| \sim \frac{M \sigma v_{\mathrm{CM}} v_{\text {orb }}}{\tilde{r} / M} .
$$

Here $M \sigma=2 \times 10^{-7}$, the extraction radius is $\tilde{r} / M \sim 10^{2}$, and $v_{\text {orb }}$ is the orbital velocity. The maximum recoil velocity from a nonspinning $\mathrm{BH}$ binary is of order $v_{\mathrm{CM}} \sim 7 \cdot 10^{-4}\left[120\right.$, so $\left|\dot{\overrightarrow{\mathcal{D}}}_{\text {recoil }} / \tilde{r}\right| \lesssim 10^{-12}$ even if $v_{\text {orb }}$ approaches unity. This contribution to the dipole radiation is way too small to account for a significant portion of the drift seen in our simulations.

Finally, a frame-dragging effect can also contribute with a nonzero drift for the scalar field. The coalescing binary drags the inertial frames, inducing a local rotation of the coordinate lines. This induces, near the binary, an apparent rotation in the $y-z$ plane of the extracted scalar field, which determines a nonvanishing real part of $\phi_{11}$. While the order of magnitude of this effect is roughly consistent with our numerical findings, the decay of the frame-dragging effect with extraction radius is not consistent with our data. Therefore frame dragging is not a dominant contribution to the observed drift.

While the DC component accounts for the order of magnitude of the drift observed in our numerical simulations, most likely the observed drift is due to a combi- nation of the effects mentioned above, and possibly others. In particular, by imposing constant-gradient boundary conditions at finite distance from the binary during the evolution we are effectively injecting energy into the system. This causes a growth of the scalar field, which may contribute significantly to the drift. This expectation should be confirmed by longer simulations and/or by simulations where the "plates" generating the scalar gradient are located further away from the binary. We hope to return to this problem in future work.

\section{CONCLUSIONS AND OUTLOOK}

We have investigated $\mathrm{BH}$ dynamics in external field profiles, by considering the very simple example of a constant scalar-field gradient. The broad features of our analysis should translate to other, more general settings, at least as long as the external force varies on length- or time-scales which are larger than the typical binary separation. Our results are in agreement with linear or slowmotion expansions, and show conclusively that black hole binaries evolving in a nontrivial background produce interesting scalar and gravitational-field dynamics.

As discussed in Appendix D, the scalar-field profiles currently considered in scalar-field dark matter models correspond roughly to $M \sigma \sim 10^{-15}$ or less for a typical stellar-mass $\mathrm{BH}$ with $M=10 M_{\odot}$. Because scalar radiation is proportional to the gradient, the experimental relevance of our setting for gravitational radiation from $\mathrm{BH}$ binaries, as observable by Advanced LIGO or similar instruments, seems negligible. However, it is interesting that gravitational-wave observations may yield upper bounds on scalar field gradients at all. Furthermore, our estimates in Appendix D predict larger field gradients for supermassive black holes with $M=10^{9} M_{\odot}$; for such binary systems the gradient could reach values as large as $M \sigma \sim 10^{-7}$, close to the values studied in this work. Finally, strong field gradients can be encountered in other - albeit more speculative - dark matter configurations, such as supermassive boson stars 99, so the possibility to come across this type of signals should be seriously taken into account. The remarkable agreement we find between our numerical results and linearized predictions indicates that the relatively small values of $M \sigma$ considered in our simulations fall into an effectively linear regime. For the more speculative scenarios leading to $M \sigma$ significantly larger than the value $2 \times 10^{-7}$ considered in Sec. VB, we therefore expect stronger numerical, nonlinear effects to be present in the radiation.

Our analysis answers some questions and sparks many new ones: why exactly do large gradients develop instabilities? Are these instabilities of a physical or purely numerical nature? Even isolated BHs moving in a scalar field should accrete: how can we understand the details of this accretion process? Another interesting question concerns spinning BHs. The original linearized analysis by Press 102 shows that Kerr BHs should in principle 
align their rotation axis with the field gradient over long enough timescales. Numerical simulations of this alignment and of its nonlinear development are an interesting (but numerically challenging) open problem, which probably requires much longer simulations than those presented in this work.

\section{ACKNOWLEDGMENTS}

E.B. and M.H. are supported by NSF CAREER Grant No. PHY-1055103. V.C. acknowledges partial financial support provided under the European Union's FP7 ERC Starting Grant "The dynamics of black holes: testing the limits of Einstein's theory" grant agreement no. DyBHo-256667, the NRHEP 295189 FP7-PEOPLE2011-IRSES Grant, and FCT-Portugal through projects PTDC/FIS/116625/2010, CERN/FP/116341/2010 and CERN/FP/123593/2011. Research at Perimeter Institute is supported by the Government of Canada through Industry Canada and by the Province of Ontario through the Ministry of Economic Development and Innovation. U.S. acknowledges support from FP7-PEOPLE2011-CIG Grant No. 293412 CBHEO, the STFC GR Roller Grant No. ST/I002006/1, the Ramón y Cajal Programme and Grant FIS2011-30145-C03-03 of the Ministry of Education and Science of Spain. Computations were performed on the "Baltasar Sete-Sois" cluster at IST, the cane cluster in Poland through PRACE DECI-7 "Black hole dynamics in metric theories of gravity", on Altamira in Cantabria through BSC grant AECT-2012-3-0012, on Caesaraugusta in Zaragoza through BSC grants AECT-2012-2-0014 and AECT2012-3-0011, XSEDE clusters SDSC Trestles and NICS Kraken through NSF Grant No. PHY-090003, Finis Terrae through Grant CESGA-ICTS-234 and the COSMOS supercomputer, part of the DiRAC HPC Facility which is funded by STFC and BIS. We thank Andrey Kaliazin for computational support and technical advice, and Ivan Stefanov for drawing our attention to references [76] 81 .

\section{Appendix A: Boosted black-hole background}

In this Appendix we shall consider the solution of the Klein-Gordon equation in a boosted BH background, i.e., in the presence of a Schwarzschild BH moving with constant velocity $v$ in the direction orthogonal to the charged planes. We shall show that this solution does not emit scalar radiation: only accelerated BHs moving in a uniform scalar-field gradient can emit scalar radiation.

To this aim, we shall first consider the (regular) solution describing a scalar field on a Schwarzschild background, generated by an infinite plane moving with velocity $v$ along the direction $z$ orthogonal to the plane. Then, we shall boost back this solution, to obtain a moving $\mathrm{BH}$ and a scalar field generated by a fixed plane.
The expression

$$
\varphi=2 \pi \sigma \gamma\left[(r-M) \cos \theta+2 M v\left(\frac{V-r}{2 M}-\log \frac{r}{2 M}\right)\right]
$$

describes a solution, regular at the horizon, of the KleinGordon equation 14 on a Schwarzschild background. Here $r_{*}=r+2 M \log (r / 2 M-1)$ is the tortoise coordinate, $V=t+r_{*}$ is the standard advanced time coordinate, $v$ is a velocity parameter and $\gamma=\left(1-v^{2}\right)^{-1 / 2}$. For $v=$ 0 , the previous expression reduces to the static solution (16). For finite $v$ and at asymptotically large distances it reduces to

$$
\varphi \sim 2 \pi \sigma \gamma(z+v t)
$$

which is related to the asymptotic solution (16) by a simple boost. Therefore, Eq. A1 describes a scalar field (on a Schwarzschild background) generated by an infinite plane moving with velocity $-v$ along $z$. We shall now boost Eq. A1 and the Schwarzschild metric, in order to find a solution of the field equations describing a $\mathrm{BH}$ moving with velocity $v$ in a field generated by an infinite charged plane at rest. The boost is more easily performed in isotropic coordinates $(t, \hat{r}, \theta, \phi)$, in which

$$
\begin{gathered}
\varphi=2 \pi \sigma \gamma\left[z\left(1+\frac{M^{2}}{4 \hat{r}^{2}}\right)+t v\right. \\
\left.-2 M v \log \frac{4+M / \hat{r}+4 \hat{r} / M}{-4+M / \hat{r}+4 \hat{r} / M}\right] .
\end{gathered}
$$

If we apply a Lorentz boost along the $z$-direction

$$
\begin{aligned}
& \bar{t}=\gamma(t+v z) \\
& \bar{z}=\gamma(z+v t) \\
& \bar{x}=x \\
& \bar{y}=y
\end{aligned}
$$

we get

$$
\begin{aligned}
\varphi & =2 \pi \sigma\left[\bar{z}+\frac{\gamma^{2} M^{2}}{4 \hat{r}^{2}}(\bar{z}-v \bar{t})\right. \\
& \left.-2 M v \gamma \log \frac{4+M / \hat{r}+4 \hat{r} / M}{-4+M / \hat{r}+4 \hat{r} / M}\right] .
\end{aligned}
$$

Note that the isotropic radial coordinate reads $\hat{r}^{2}=\bar{x}^{2}+$ $\bar{y}^{2}+\gamma^{2}(\bar{z}-v \bar{t})^{2}$.

Along the $\bar{z}$-axis, this expression has the form

$$
\varphi \sim 2 \pi \sigma\left[\bar{z}+\frac{M^{2}(1-16 v)}{4 \bar{z}}+\frac{M^{2}(1-16 v) v \bar{t}}{4 \bar{z}^{2}}\right]+\mathcal{O}\left(\bar{z}^{-3}\right) .
$$

Introducing polar coordinates in the boosted frame

$$
\begin{aligned}
& \bar{x}=\bar{r} \sin \bar{\theta} \cos \bar{\phi}, \\
& \bar{y}=\bar{r} \sin \bar{\theta} \sin \bar{\phi}, \\
& \bar{z}=\bar{r} \cos \bar{\theta},
\end{aligned}
$$


we have (since $\left.\gamma^{2}-1=v^{2} \gamma^{2}\right)$

$$
\hat{r}=\sqrt{\bar{r}^{2}\left(1+v^{2} \gamma^{2} \cos \bar{\theta}\right)-2 v \gamma^{2} \bar{t} \bar{r} \cos \bar{\theta}+\gamma^{2} v^{2} \bar{t}^{2}}
$$

If we assume small boosts $v \ll 1$, so we can neglect terms $O\left(v^{2}\right), \gamma \simeq 1$. We can also assume that $v \bar{t} \ll \bar{r}$, i.e., that $v \bar{t} / \bar{r} \ll 1$, even though this quantity can be larger than $v^{2}$. Expanding Eq. A8 in these two dimensionless quantities, up to first order in $v^{2}$ and second order in $v \bar{t} / \bar{r} \ll 1$ ), we find

$$
\hat{r} \simeq \bar{r}\left[1-\left(\frac{v \bar{t}}{\bar{r}}\right) \cos \bar{\theta}+\frac{1}{2}\left(\frac{v \bar{t}}{\bar{r}}\right)^{2}\right] .
$$

Therefore

$$
\begin{aligned}
\varphi & =2 \pi \sigma\left[\bar{r} \cos \bar{\theta}+\frac{M^{2}}{4 \bar{r}}\left(\cos \bar{\theta}+2\left(\frac{v \bar{t}}{\bar{r}}\right) \cos ^{2} \bar{\theta}\right)\right. \\
& \left.-\frac{4 M^{2} v}{\bar{r}}\left(1+\left(\frac{v \bar{t}}{\bar{r}}\right) \cos \bar{\theta}\right)\right]+O\left(\frac{1}{\bar{r}^{3}}\right) \\
& =2 \pi \sigma\left[\bar{r} \cos \bar{\theta}+\frac{M^{2}}{4 \bar{r}}(\cos \bar{\theta}-16 v)\right. \\
& \left.+\frac{M^{2} v \bar{t}}{2 \bar{r}^{2}} \cos \bar{\theta}(\cos \bar{\theta}-8 v)\right]
\end{aligned}
$$

As discussed in 88], we find that the scalar charge is the (spherically symmetric component of the) coefficient of $1 / \bar{r}$ in this expansion, divided by $2 M$, thus

$$
Q=-4 \pi \sigma M v
$$

Furthermore, the scalar-field multipoles are

$$
\begin{aligned}
\mathcal{M} & =8 \pi \sigma M^{2} v, \\
\dot{\overrightarrow{\mathcal{D}}} & =-\frac{\pi \sigma M^{2}}{2} \hat{z} .
\end{aligned}
$$

All of these quantities are constant in time, therefore there is no emitted scalar radiation. We can conclude that if a $\mathrm{BH}$ moves with constant velocity in a scalarfield gradient, there is no scalar emission; in order to get scalar radiation the $\mathrm{BH}$ should have a nonvanishing acceleration, as shown analytically in Section IIIB and numerically in Section $\mathrm{VB}$.

\section{Appendix B: Approximate solution for monopole and dipole radiation from a quasi-circular binary in a scalar gradient}

This Appendix completes the approximate solution discussed in Section IIIB2, which describes the scalar field generated by a binary BH in quasicircular orbit in a scalar-field gradient. The center-of-mass acceleration is:

$$
\begin{aligned}
\vec{a}_{\mathrm{CM}}= & \frac{4 a^{(0)} \dot{\chi}^{(0)}}{5 \tau_{\mathrm{q}}} \frac{(q-1)}{(q+1)}\left[\hat{z} \cos \chi^{(0)}-\hat{y} \sin \chi^{(0)}\right],(\mathrm{B} 1) \\
\vec{v}_{\mathrm{CM}}= & \frac{4 a^{(0)}}{5 \tau_{\mathrm{q}}} \frac{(q-1)}{(q+1)}\left[\hat{y}\left(\cos \chi^{(0)}-1\right)+\hat{z} \sin \chi^{(0)}\right] \\
& +\vec{V}_{0}, \\
\vec{z}_{\mathrm{CM}}= & \frac{4 a^{(0)}}{5 \tau_{\mathrm{q}} \dot{\chi}^{(0)}} \frac{(q-1)}{(q+1)}\left[\hat{y} \sin \chi^{(0)}-\hat{z}\left(\cos \chi^{(0)}-1\right)\right] \\
& +\vec{V}_{0}\left(t-t_{0}\right),
\end{aligned}
$$

where $q=M_{1} / M_{2}$ is the mass ratio, $\chi^{(0)}(t)=\dot{\chi}^{(0)} t+\psi_{0}$ is the zeroth-order orbital phase, which vanishes at $t=t_{0}$, and $\vec{V}_{0}=\vec{v}_{\mathrm{CM}}\left(t=t_{0}\right)$ is the initial velocity of the center of mass relative to the scalar gradient. Without loss of generality, the choice $\vec{z}_{\mathrm{CM}}\left(t=t_{0}\right)=0$ has been made. Note that in the equal-mass limit $(q \rightarrow 1)$, the center-ofmass recoil vanishes, on account of symmetry.

Given the scalar charges

$$
\begin{aligned}
Q_{1} & =\frac{8 \pi \sigma M_{1}^{2}}{M}\left(M \vec{v}_{\mathrm{CM}} \cdot \hat{z}+M_{2}[\dot{a} \sin \chi+a \dot{\chi} \cos \chi]\right), \\
Q_{2} & =\frac{8 \pi \sigma M_{2}^{2}}{M}\left(M \vec{v}_{\mathrm{CM}} \cdot \hat{z}-M_{1}[\dot{a} \sin \chi+a \dot{\chi} \cos \chi]\right),
\end{aligned}
$$

the monopole component of the scalar field is:

$$
\mathcal{M}=Q_{1}+Q_{2}=\frac{8 \pi \sigma}{M}\left(M\left(M_{1}^{2}+M_{2}^{2}\right)\left(\vec{v}_{\mathrm{CM}} \cdot \hat{z}\right)+M_{1} M_{2}\left(M_{1}-M_{2}\right)[\dot{a} \sin \chi+a \dot{\chi} \cos \chi]\right),
$$

while the dipole component reads

$$
\overrightarrow{\mathcal{D}}=Q_{1} \vec{z}_{1}(t)+Q_{2} \vec{z}_{2}(t)=\overrightarrow{\mathcal{D}}_{\mathrm{CM}}+\overrightarrow{\mathcal{D}}_{\mathrm{rel}, \mathrm{DC}}+\overrightarrow{\mathcal{D}}_{\mathrm{rel}, \mathrm{osc}}
$$

with

$$
\begin{aligned}
\overrightarrow{\mathcal{D}}_{\mathrm{CM}} & =\frac{8 \pi \sigma M_{1} M_{2}\left(M_{1}-M_{2}\right) a}{M}\left(\vec{z}_{\mathrm{CM}} \dot{\chi} \cos \chi+\left(\vec{v}_{\mathrm{CM}} \cdot \hat{z}\right)(\hat{y} \cos \chi+\hat{z} \sin \chi)\right), \\
\overrightarrow{\mathcal{D}}_{\mathrm{rel}, \mathrm{DC}} & =\frac{8 \pi \sigma M_{1}^{2} M_{2}^{2} a}{M^{2}}(\dot{a} \hat{z}+a \dot{\chi} \hat{y}), \\
\overrightarrow{\mathcal{D}}_{\text {rel, osc }} & =\frac{8 \pi \sigma M_{1}^{2} M_{2}^{2} a}{M^{2}}(\dot{a}[\hat{y} \sin (2 \chi)-\hat{z} \cos (2 \chi)]+a \dot{\chi}[\hat{y} \cos (2 \chi)+\hat{z} \sin (2 \chi)]) .
\end{aligned}
$$


From the previous expression we find

$$
\dot{\overrightarrow{\mathcal{D}}}=\dot{\overrightarrow{\mathcal{D}}}_{\mathrm{CM}}+\dot{\overrightarrow{\mathcal{D}}}_{\mathrm{rel}, \mathrm{DC}}+\dot{\overrightarrow{\mathcal{D}}}_{\mathrm{rel}, \text { osc }}
$$

with

$$
\begin{aligned}
& \dot{\overrightarrow{\mathcal{D}}}_{\mathrm{CM}}=\frac{8 \pi \sigma M_{1} M_{2}\left(M_{1}-M_{2}\right) a}{M}\left(\dot{\chi}\left(\vec{v}_{\mathrm{CM}} \cos \chi-\vec{z}_{\mathrm{CM}} \dot{\chi} \sin \chi\right)+\left(\vec{a}_{\mathrm{CM}} \cdot \hat{z}\right)(\hat{y} \cos \chi+\hat{z} \sin \chi)\right. \\
& \left.+\left(\vec{v}_{\mathrm{CM}} \cdot \hat{z}\right) \dot{\chi}(\hat{z} \cos \chi-\hat{y} \sin \chi)\right), \\
& \dot{\overrightarrow{\mathcal{D}}}_{\mathrm{rel}, \mathrm{DC}}=\frac{8 \pi \sigma M_{1}^{2} M_{2}^{2} a}{M^{2}}(\ddot{a} \hat{z}+(2 \dot{a} \dot{\chi}+a \ddot{\chi}) \hat{y}) \\
& \dot{\overrightarrow{\mathcal{D}}}_{\text {rel, osc }}=\frac{8 \pi \sigma M_{1}^{2} M_{2}^{2} a}{M^{2}}\left(\left(\ddot{a}-2 a \dot{\chi}^{2}\right)[\hat{y} \sin (2 \chi)-\hat{z} \cos (2 \chi)]+(4 \dot{a} \dot{\chi}+a \ddot{\chi})[\hat{y} \cos (2 \chi)+\hat{z} \sin (2 \chi)]\right) \text {, }
\end{aligned}
$$

where $M=M_{1}+M_{2}$ is the total mass, $\vec{v}_{\mathrm{CM}}$ and $\vec{a}_{\mathrm{CM}}$ are the velocity and acceleration of the center of mass, respectively, and terms of order higher than $1 / c^{5}$ in the dipole moment have been dropped. If we neglect radiative effects, and express the dipole waveform using the $\ell, m$ multipole components introduced in (72), we find

$$
\begin{aligned}
\varphi_{10} & =0, \\
\varphi_{1 \pm 1} & =i A e^{\mp 2 i \chi}+\text { gradient term },
\end{aligned}
$$

where the amplitude of oscillation is given by

$$
A=\sqrt{\frac{512 \pi^{3}}{3}}(M \sigma) \frac{M}{r} \nu^{2}(M \dot{\chi})^{2 / 3},
$$

and $\nu=M_{1} M_{2} / M^{2}$ is the symmetric mass ratio. For our simulation with $q=3$ and $M \sigma=2 \times 10^{-7}$ we find that $M \dot{\chi} \sim 2 \times 10^{-2}$, and therefore the theoretical prediction for the dipole amplitude is $A \sim 4 \times 10^{-10}$. This is in order-of-magnitude agreement with the observed dipole radiation in our numerical simulations.

\section{Appendix C: 3+1 Evolution equations}

In terms of the BSSN variables defined in Eq. (65), the scalar field $\varphi$ and the scalar curvature $K_{\varphi}$ defined in Eq. 44, the BSSN evolution equations are given by

$$
\begin{aligned}
\partial_{t} \tilde{\gamma}_{i j}= & \beta^{m} \partial_{m} \tilde{\gamma}_{i j}+2 \tilde{\gamma}_{m(i} \partial_{j)} \beta^{m}-\frac{2}{3} \tilde{\gamma}_{i j} \partial_{m} \beta^{m}-2 \alpha \tilde{A}_{i j} \\
\partial_{t} \chi= & \beta^{m} \partial_{m} \chi+\frac{2}{3} \chi\left(\alpha K-\partial_{m} \beta^{m}\right) \\
\partial_{t} \tilde{A}_{i j}= & \beta^{m} \partial_{m} \tilde{A}_{i j}+2 \tilde{A}_{m(i} \partial_{j)} \beta^{m}-\frac{2}{3} \tilde{A}_{i j} \partial_{m} \beta^{m}+\chi\left(\alpha R_{i j}-D_{i} D_{j} \alpha\right)^{\mathrm{TF}}+\alpha\left(K \tilde{A}_{i j}-2 \tilde{A}_{i}{ }^{m} \tilde{A}_{m j}\right) \\
& -\alpha \chi\left(\partial_{i} \varphi \partial_{j} \varphi-\frac{1}{3} \tilde{\gamma}_{i j} \tilde{\gamma}^{m n} \partial_{m} \varphi \partial_{n} \varphi\right) \\
\partial_{t} K= & \beta^{m} \partial_{m} K-D^{m} D_{m} \alpha+\alpha\left(\tilde{A}^{m n} \tilde{A}_{m n}+\frac{1}{3} K^{2}\right)+4 \alpha K_{\varphi}, \\
\partial_{t} \tilde{\Gamma}^{i}= & \beta^{m} \partial_{m} \tilde{\Gamma}^{i}-\tilde{\Gamma}^{m} \partial_{m} \beta^{i}+\frac{2}{3} \tilde{\Gamma}^{i} \partial_{m} \beta^{m}+2 \alpha \tilde{\Gamma}_{m n}^{i} \tilde{A}^{m n}+\frac{1}{3} \tilde{\gamma}^{i m} \partial_{m} \partial_{n} \beta^{n}+\tilde{\gamma}^{m n} \partial_{m} \partial_{n} \beta^{i} \\
& -\frac{4}{3} \alpha \tilde{\gamma}^{i m} \partial_{m} K-\tilde{A}^{i m}\left(3 \alpha \frac{\partial_{m} \chi}{\chi}+2 \partial_{m} \alpha\right)-\frac{2}{3}\left(\tilde{\Gamma}^{i}-\tilde{\gamma}^{m n} \tilde{\Gamma}_{m n}^{i}\right) \partial_{k} \beta^{k}-4 K_{\varphi} \tilde{\gamma}^{i m} \partial_{m} \varphi
\end{aligned}
$$

Likewise, we write the Hamiltonian and momentum constraints in terms of the BSSN variables as

$$
\begin{aligned}
& { }^{(3)} R+\frac{2}{3} K^{2}-\tilde{\gamma}^{m n} \tilde{\gamma}^{k l} \tilde{A}_{m k} \tilde{A}_{n l}=4 K_{\varphi}^{2}+\partial_{i} \varphi \partial^{i} \varphi \\
& \tilde{D}_{m} \tilde{A}_{i}^{m}-\frac{2}{3} \partial_{i} K-\frac{3}{2 \chi} \tilde{A}_{i}^{m} \partial_{m} \chi=2 K_{\varphi} \partial_{i} \varphi
\end{aligned}
$$

The lapse function and shift vector are evolved as in the case of vacuum general relativistic $\mathrm{BH}$ simulations (cf. [121]) according to

$$
\begin{aligned}
\partial_{t} \alpha & =\beta^{m} \partial_{m} \alpha-2 \alpha K \\
\partial_{t} \beta^{i} & =\beta^{m} \partial_{m} \beta^{i}+\frac{3}{4} \tilde{\Gamma}^{i}-\eta \beta^{i} .
\end{aligned}
$$


Following [122], we use a position-dependent parameter $\eta$; specifically, we set

$$
\eta=\eta_{0} \frac{R^{2}}{r^{2}+R^{2}} \frac{\left|\vec{r}_{1}\right|+\left|\vec{r}_{2}\right|}{2\left(M_{1}\left|\vec{r}_{1}\right|+M_{2}\left|\vec{r}_{2}\right|\right)},
$$

where $r$ is the coordinate distance from the origin, $\vec{r}_{1,2}$ are the position vectors from either hole and $M_{1,2}$ are the $\mathrm{BH}$ masses. Lapse and shift are initialized as $\alpha=\sqrt{\chi}$ and $\beta^{i}=0$, respectively.

\section{Appendix D: Order of magnitude of the scalar gradient in a cosmological scenario}

Scalar fields on galactic scales have been considered by many authors as a possible explanation for the rotation curves in galaxies and as alternatives to cold dark matter [89 97] (see also [98). The aim of this Appendix is to estimate the typical magnitudes of the scalar-field gradients predicted by these models.

Sadeghian et al. [123] recently studied the distribution of dark matter around massive BHs in full general relativity using a Hernquist profile [124, which is a good description of isolated dark matter halos [125. According to 123 , a typical density for the dark matter halo is $\rho \sim 10^{10} \mathrm{Gev} / \mathrm{cm}^{3}$. One should be very cautious in comparing our stationary, free scalar field configuration with those suggested by cosmological models. Indeed, in many of the works cited above the scalar field is rapidly oscillating, and the mass term and the potential always play a role. In order to estimate the order of magnitude of the scalar-field gradient we can simply note that, neglecting the contribution of the potential $V(\phi)$ and restoring physical units, the mass-energy density is of the order (see e.g. 92])

$$
G \rho \sim\left|\phi_{, t}\right|^{2}+c^{2}\left|\phi_{, r}\right|^{2},
$$

therefore the gradient $\sigma=\phi_{, r}$ is at most

$$
\sigma \sim \sqrt{\frac{G \rho}{c^{2}}} \sim 10^{-16} \mathrm{~km}^{-1} \sim \frac{10^{-15}}{10 M_{\odot}} .
$$

In our simulations we set the $\mathrm{BH}$ mass $M=1$. For a stellar-mass $\mathrm{BH}\left(M=10 M_{\odot}\right)$ moving near the galactic center a typical scalar-field gradient is therefore $M \sigma \sim$ $10^{-15}$; for a supermassive $\mathrm{BH}\left(M=10^{9} M_{\odot}\right)$, a typical gradient would be $M \sigma \sim 10^{-7}$, the same order of magnitude studied in this paper. This should be considered as a rough upper limit: in scenarios in which the scalar field is rapidly oscillating the kinetic term should contribute to the energy density more than the gradient.
[1] H. Goenner, Gen.Rel.Grav. 44, 2077 (2012) arXiv:1204.3455 [gr-qc]

[2] C. H. Brans, (2005), arXiv:gr-qc/0506063 [gr-qc]

[3] T. Singh and L. Rai, Gen.Rel.Grav. 15, 875 (1983).

[4] Y. Fujii and K. Maeda, The scalar-tensor theory of gravitation (Cambridge University Press, Cambridge, England, 2003).

[5] V. Faraoni, Cosmology in Scalar-Tensor Gravity (Springer, 2004).

[6] M. S. Berman, Introduction to General Relativistic and Scalar-tensor Cosmologies (Nova Science Pub Inc, 2007).

[7] C. Brans and R. Dicke, Phys.Rev. 124, 925 (1961)

[8] R. H. Dicke, Phys. Rev. 125, 2163 (1962).

[9] C. Brans, Phys.Rev. 125, 2194 (1962)

[10] P. G. Bergmann, Int.J.Theor.Phys. 1, 25 (1968).

[11] R. V. Wagoner, Phys.Rev. D1, 3209 (1970).

[12] T. Damour and G. Esposito-Farese, Class.Quant.Grav. 9, $2093(1992)$

[13] T. Chiba, T. Harada, and K.-i. Nakao, Prog.Theor.Phys.Suppl. 128, 335 (1997)

[14] C. Will, Theory and experiment in gravitational physics (Cambridge University Press, Cambridge, England, 1993).

[15] C. M. Will, Living Rev. Relativity 9 (2005), arXiv:grqc/0510072

[16] T. Clifton, P. G. Ferreira, A. Padilla, and C. Skordis, Phys.Rept. 513, 1 (2012), arXiv:1106.2476 [astroph.CO]

[17] C. M. Will and H. W. Zaglauer, Astrophys.J. 346, 366
(1989)

18] T. Damour and G. Esposito-Farese, Phys.Rev.Lett. 70, $2220(1993)$

[19] T. Damour and G. Esposito-Farese, Phys.Rev. D53, 5541 (1996), arXiv:gr-qc/9506063 [gr-qc]

[20] T. Damour and G. Esposito-Farese, Phys.Rev. D54, 1474 (1996), arXiv:gr-qc/9602056 [gr-qc]

[21] T. Damour and G. Esposito-Farese, Phys.Rev. D58, 042001 (1998), arXiv:gr-qc/9803031 [gr-qc]

[22] T. Damour, (2007), arXiv:0704.0749 [gr-qc]

[23] N. R. Bhat, M. Bailes, and J. P. Verbiest, Phys.Rev. D77, 124017 (2008), arXiv:0804.0956 [astro-ph]

[24] K. Lazaridis, N. Wex, A. Jessner, M. Kramer, B. Stappers, et al., (2009), arXiv:0908.0285 [astro-ph.GA]

[25] G. Esposito-Farese, Fundam.Theor.Phys. 162, 461 (2011), arXiv:0905.2575 [gr-qc]

[26] J. Alsing, E. Berti, C. M. Will, and H. Zaglauer, Phys.Rev. D85, 064041 (2012), arXiv:1112.4903 [gr-qc]

[27] P. C. Freire, N. Wex, G. Esposito-Farese, J. P. Verbiest, M. Bailes, et al., Mon.Not.Roy.Astron.Soc. 423, 3328 (2012), arXiv:1205.1450 [astro-ph.GA]

[28] C. M. Will, Phys.Rev. D50, 6058 (1994), arXiv:grqc/9406022 [gr-qc].

[29] P. D. Scharre and C. M. Will, Phys.Rev. D65, 042002 (2002), arXiv:gr-qc/0109044 [gr-qc].

[30] C. M. Will and N. Yunes, Class.Quant.Grav. 21, 4367 (2004), arXiv:gr-qc/0403100 [gr-qc]

[31] E. Berti, A. Buonanno, and C. M. Will, Phys. Rev. D71, 084025 (2005), arXiv:gr-qc/0411129.

[32] E. Berti, A. Buonanno, and C. M. Will, Class. Quantum 
Grav. 22, S943 (2005), arXiv:gr-qc/0504017

[33] K. Yagi and T. Tanaka, Phys.Rev. D81, 064008 (2010). arXiv:0906.4269 [gr-qc]

[34] N. Yunes, P. Pani, and V. Cardoso, Phys.Rev. D85, 102003 (2012), arXiv:1112.3351 [gr-qc]

[35] E. Berti, L. Gualtieri, M. Horbatsch, and J. Alsing, Phys.Rev. D85, 122005 (2012), arXiv:1204.4340 [gr-qc]

[36] A. Arvanitaki and S. Dubovsky, Phys.Rev. D83, 044026 (2011), arXiv:1004.3558 [hep-th]

[37] S. Dubovsky and V. Gorbenko, Phys.Rev. D83, 106002 (2011), arXiv:1012.2893 [hep-ph].

[38] H. Yoshino and H. Kodama, Prog.Theor.Phys. 128, 153 (2012), arXiv:1203.5070 [gr-qc]

[39] H. Witek, V. Cardoso, A. Ishibashi, and U. Sperhake, (2012), arXiv:1212.0551 [gr-qc]

[40] A. Arvanitaki, S. Dimopoulos, S. Dubovsky, N. Kaloper, and J. March-Russell, Phys.Rev. D81, 123530 (2010), arXiv:0905.4720 [hep-th].

[41] V. Cardoso, S. Chakrabarti, P. Pani, E. Berti, and L. Gualtieri, Phys.Rev.Lett. 107, 241101 (2011), arXiv:1109.6021 [gr-qc].

[42] S. Hawking, Commun.Math.Phys. 25, 167 (1972).

[43] K. S. Thorne and J. J. Dykla, Astrophys. J. Lett. 166, L35 (1971).

[44] J. Chase, Commun.Math.Phys. 19, 276 (1970).

[45] M. Heusler, Class.Quant.Grav. 12, 2021 (1995). arXiv:gr-qc/9503053 [gr-qc]

[46] T. P. Sotiriou and V. Faraoni, Phys.Rev.Lett. 108, 081103 (2012), arXiv:1109.6324 [gr-qc]

[47] M. A. Scheel, S. L. Shapiro, and S. A. Teukolsky, Phys.Rev. D51, 4208 (1995), arXiv:gr-qc/9411025 [grqc],

[48] M. A. Scheel, S. L. Shapiro, and S. A. Teukolsky, Phys.Rev. D51, 4236 (1995), arXiv:gr-qc/9411026 [grqc],

[49] M. Shibata, K. Nakao, and T. Nakamura, Phys.Rev. D50, 7304 (1994).

[50] T. Harada, T. Chiba, K.-i. Nakao, and T. Nakamura, Phys.Rev. D55, 2024 (1997), arXiv:gr-qc/9611031 [grqc].

[51] J. Novak, Phys.Rev. D57, 4789 (1998), arXiv:grqc/9707041 [gr-qc].

[52] J. Kerimo and D. Kalligas, Phys.Rev. D58, 104002 (1998).

[53] D. Psaltis, D. Perrodin, K. R. Dienes, and I. Mocioiu, Phys.Rev.Lett. 100, 091101 (2008), arXiv:0710.4564 [astro-ph]

[54] J. Kerimo, Phys.Rev. D62, 104005 (2000).

[55] E. Barausse and T. P. Sotiriou, Phys.Rev.Lett. 101, 099001 (2008), arXiv:0803.3433 [gr-qc].

[56] E. Berti, (2013), arXiv:1302.5702 [gr-qc]

[57] J. D. Bekenstein, (1996), arXiv:gr-qc/9605059 [gr-qc]

[58] P. T. Chrusciel, J. L. Costa, and M. Heusler, Living Rev.Rel. 15, 7 (2012), arXiv:1205.6112 [gr-qc]

[59] T. Jacobson, Phys.Rev.Lett. 83, 2699 (1999), arXiv:astro-ph/9905303 [astro-ph]

[60] K. Yagi, N. Yunes, and T. Tanaka, Phys.Rev. D86, 044037 (2012), arXiv:1206.6130 [gr-qc]

[61] N. Yunes and F. Pretorius, Phys.Rev. D79, 084043 (2009), arXiv:0902.4669 [gr-qc]

[62] K. Konno, T. Matsuyama, and S. Tanda, Prog.Theor.Phys. 122, 561 (2009), arXiv:0902.4767 [gr-qc].

[63] N. Yunes and L. C. Stein, Phys.Rev. D83, 104002
(2011), arXiv:1101.2921 [gr-qc]]

[64] P. Pani, C. F. Macedo, L. C. Crispino, and V. Cardoso, Phys.Rev. D84, 087501 (2011), arXiv:1109.3996 [gr-qc].

[65] K. Yagi, L. C. Stein, N. Yunes, and T. Tanaka, Phys.Rev. D85, 064022 (2012), arXiv:1110.5950 [gr-qc]

[66] S. Droz, M. Heusler, and N. Straumann, Phys.Lett. B268, 371 (1991)

[67] M. Heusler, S. Droz, and N. Straumann, Phys.Lett. B271, 61 (1991)

[68] M. Heusler, S. Droz, and N. Straumann, Phys.Lett. B285, 21 (1992).

[69] N. Shiiki and N. Sawado, (2005), arXiv:gr-qc/0501025 [gr-qc]

[70] A. Nicolis, R. Rattazzi, and E. Trincherini, Phys.Rev. D79, 064036 (2009), arXiv:0811.2197 [hep-th]

[71] K. Van Acoleyen and J. Van Doorsselaere, Phys.Rev. D83, 084025 (2011), arXiv:1102.0487 [gr-qc]

[72] M. A. Luty, M. Porrati, and R. Rattazzi, JHEP 0309, 029 (2003), arXiv:hep-th/0303116 [hep-th]

[73] C. de Rham, G. Gabadadze, and A. J. Tolley, Phys.Rev.Lett. 106, 231101 (2011), arXiv:1011.1232 [hep-th].

[74] A. Vainshtein, Phys.Lett. B39, 393 (1972)

[75] L. Hui and A. Nicolis, (2012), arXiv:1202.1296 [hep-th]

[76] I. Z. Stefanov, S. S. Yazadjiev, and M. D. Todorov, Phys.Rev. D75, 084036 (2007), arXiv:0704.3784 [gr-qc]

[77] I. Z. Stefanov, S. S. Yazadjiev, and M. D. Todorov, Mod.Phys.Lett. A23, 2915 (2008), arXiv:0708.4141 [grqc]

[78] I. Z. Stefanov, S. S. Yazadjiev, and M. D. Todorov, (2007), arXiv:0708.3203 [gr-qc].

[79] I. Z. Stefanov, S. S. Yazadjiev, D. A. Georgieva, and M. D. Todorov, Int.J.Mod.Phys. D20, 2471 (2011), arXiv:0909.0196 [gr-qc].

[80] I. Z. Stefanov, S. S. Yazadjiev, and M. D. Todorov, Class.Quant.Grav. 26, 015006 (2009)

[81] D. D. Doneva, S. S. Yazadjiev, K. D. Kokkotas, and I. Z. Stefanov, Phys.Rev. D82, 064030 (2010), arXiv:1007.1767 [gr-qc].

[82] D. Eardley, Astrophys.J. 196, L59 (1975).

[83] S. Mirshekari and C. M. Will, (2013), arXiv:1301.4680 [gr-qc]

[84] E. Barausse, C. Palenzuela, M. Ponce, and L. Lehner, (2012), arXiv:1212.5053 [gr-qc]

[85] J. Healy, T. Bode, R. Haas, E. Pazos, P. Laguna, et al., (2011), arXiv:1112.3928 [gr-qc].

[86] K. Yagi, N. Yunes, and T. Tanaka, Phys.Rev.Lett. 109, 251105 (2012), arXiv:1208.5102 [gr-qc],

[87] K. Yagi, L. C. Stein, N. Yunes, and T. Tanaka, (2013), arXiv:1302.1918 [gr-qc],

[88] M. Horbatsch and C. Burgess, (2011), arXiv:1111.4009 [gr-qc]

[89] S.-J. Sin, Phys. Rev. D 50, 3650 (1994)

[90] W. Hu, R. Barkana, and A. Gruzinov, Phys.Rev.Lett. 85, 1158 (2000), arXiv:astro-ph/0003365 [astro-ph].

[91] T. Matos and F. S. Guzman, Class.Quant.Grav. 17, L9 (2000), arXiv:gr-qc/9810028 [gr-qc].

[92] F. E. Schunck, (1998), arXiv:astro-ph/9802258 [astro$\mathrm{ph}]$.

[93] V. Sahni and L.-M. Wang, Phys.Rev. D62, 103517 (2000), arXiv:astro-ph/9910097 [astro-ph],

[94] T. Matos, F. S. Guzman, and D. Nunez, Phys.Rev. D62, 061301 (2000), arXiv:astro-ph/0003398 [astro-ph].

[95] A. Arbey, J. Lesgourgues, and P. Salati, Phys.Rev. 
D64, 123528 (2001), arXiv:astro-ph/0105564 [astro-ph]

[96] A. Arbey, J. Lesgourgues, and P. Salati, Phys.Rev. D68, 023511 (2003), arXiv:astro-ph/0301533 [astro-ph].

[97] C. Boehmer and T. Harko, JCAP 0706, 025 (2007) arXiv:0705.4158 [astro-ph]

[98] M. Alcubierre, F. S. Guzman, T. Matos, D. Nunez, L. A. Urena-Lopez, et al., Class.Quant.Grav. 19, 5017 (2002) arXiv:gr-qc/0110102 [gr-qc].

[99] C. F. B. Macedo, P. Pani, V. Cardoso, and L. C. B. Crispino, (2013), arXiv:1302.2646 [gr-qc]

[100] D. Eardley, D. Lee, and A. Lightman, Phys.Rev. D8, 3308 (1973)

[101] D. Eardley, D. Lee, A. Lightman, R. Wagoner, and C. Will, Phys.Rev.Lett. 30, 884 (1973).

[102] W. H. Press, Astrophys. J. 175, 243 (1972).

[103] L. Blanchet, Living Rev. Relativity 9 (2006), arXiv:grqc/0202016.

[104] M. Salgado, D. M.-d. Rio, M. Alcubierre, and D. Nunez, Phys. Rev. D77, 104010 (2008), arXiv:0801.2372 [grqc].

[105] M. Zilhao, H. Witek, U. Sperhake, V. Cardoso, L. Gualtieri, C. Herdeiro, and A. Nerozzi, Phys. Rev. D81, 084052 (2010), arXiv:1001.2302 [gr-qc]

[106] M. Alcubierre, Introduction to 3+1 Numerical Relativity (Oxford University Press, Oxford, U.K., 2008).

[107] Cactus Computational Toolkit homepage: http://www . cactuscode.org/.

[108] U. Sperhake, Phys. Rev. D76, 104015 (2007), arXiv:grqc/0606079 [gr-qc].

[109] U. Sperhake, B. Brügmann, D. Müller, and C. F. Sopuerta, Class. Quantum Grav. 28, 134004 (2010), arXiv:1012.3173 [gr-qc].

[110] E. Schnetter, S. H. Hawley, and I. Hawke, Class. Quan- tum Grav. 21, 1465 (2004), gr-qc/0310042

[111] J. Thornburg, Phys. Rev. D54, 4899 (1996), grqc/9508014.

[112] J. Thornburg, Class. Quantum Grav. 21, 743 (2004) gr-qc/0306056.

[113] M. Ansorg, B. Brügmann, and W. Tichy, Phys. Rev. D70, 064011 (2004), arXiv:gr-qc/0404056 [gr-qc].

[114] C. Palenzuela, L. Lehner, and S. Yoshida, Phys.Rev. D81, 084007 (2010), arXiv:0911.3889 [gr-qc].

[115] C. Palenzuela, M. Anderson, L. Lehner, S. L. Liebling, and D. Neilsen, Phys.Rev.Lett. 103, 081101 (2009), arXiv:0905.1121 [astro-ph.HE].

[116] H. Witek, M. Zilhão, L. Gualtieri, V. Cardoso, C. Herdeiro, A. Nerozzi, and U. Sperhake, Phys. Rev. D82, 104014 (2010), arXiv:1006.3081 [gr-qc].

[117] E. Berti, V. Cardoso, J. A. González, and U. Sperhake, Phys. Rev. D75, 124017 (2007), arXiv:gr-qc/0701086

[118] E. Berti, V. Cardoso, and C. M. Will, Phys. Rev. D73, 064030 (2006), arXiv:gr-qc/0512160.

[119] E. Berti, V. Cardoso, and A. O. Starinets, Class. Quantum Grav. 26, 163001 (2009), arXiv:0905.2975 [gr-qc]

[120] J. A. González, U. Sperhake, B. Brügmann, M. D. Hannam, and S. Husa, Phys. Rev. Lett. 98, 091101 (2007), gr-qc/0610154.

[121] J. R. van Meter, J. G. Baker, M. Koppitz, and D.-I. Choi, Phys. Rev. D73, 124011 (2006), gr-qc/0605030.

[122] E. Schnetter, Class. Quantum Grav. 27, 167001 (2010), arXiv:1003.0859 [gr-qc].

[123] L. Sadeghian, F. Ferrer, and C. M. Will, (2013), arXiv:1305.2619 [astro-ph.GA]

[124] L. Hernquist, Astrophys.J. 356, 359 (1990).

[125] E. Visbal, A. Loeb, and L. Hernquist, (2012), arXiv:1206.5852 [astro-ph.CO]. 